\title{
Brief communication: Interest of a regional climate model against ERA5 to simulate the near-surface climate of the Greenland ice sheet
}

\author{
Alison Delhasse ${ }^{1}$, Christoph Kittel ${ }^{1}$, Charles Amory ${ }^{1}$, Stefan Hofer ${ }^{1,2}$, and Xavier Fettweis ${ }^{1}$ \\ ${ }^{1}$ Laboratory of Climatology, Department of Geography, SPHERES, University of Liège, Liège, Belgium \\ ${ }^{2}$ School of Geographical Sciences, University of Bristol, Bristol, UK \\ Correspondence to: Alison Delhasse (alison.delhasse@uliege.be)
}


Table S1. Dismissed AWS per studied variable (2-m temperature, 10-m wind speed, longwave and shortwave downward radiative flux) and justifications.

\begin{tabular}{|c|c|c|}
\hline Variable & AWS & Justification \\
\hline & SCO_L & Difference between AWS and interpolated model elevations higher than $250 \mathrm{~m}$ in absolute value for all models \\
\hline & TAS_A & exemple fev et mars 2014: obs » par rapport a MAR (voir resume station) \\
\hline Surface & & Defavourable statistic comparisons (correlation, RMSE and RMSEc) for the 5 simulations* \\
\hline \multirow[t]{4}{*}{ Pressure } & QAS_A & Defavourable statistic comparisons (correlation, RMSE and RMSEc) for the 5 simulations* \\
\hline & TAS_L & Defavourable statistic comparisons (correlation, RMSE and RMSEc) for the 5 simulations* \\
\hline & SCO_L & Difference between AWS and interpolated model elevations higher than $250 \mathrm{~m}$ in absolute value for all models \\
\hline & TAS_A & Defavourable statistic comparisons (correlation, RMSE and RMSEc) for the 5 simulations* \\
\hline \multirow[t]{3}{*}{$\mathrm{T} 2 \mathrm{M}$} & NUK_L & Defavourable statistic comparisons (correlation, RMSE and RMSEc) for the 5 simulations* \\
\hline & TAS_L & Defavourable statistic comparisons (correlation, RMSE and RMSEc) for the 5 simulations* \\
\hline & SCO_L & Difference between AWS and interpolated model elevations higher than $250 \mathrm{~m}$ in absolute value for all models \\
\hline Wind & SCO_U & Defavourable statistic comparisons (correlation, RMSE and RMSEc) for the 5 simulations* \\
\hline \multirow[t]{2}{*}{ Speed } & QAS_A & Defavourable statistic comparisons (correlation, RMSE and RMSEc) for the 5 simulations* \\
\hline & NUK_L & Defavourable statistic comparisons (correlation, RMSE and RMSEc) for the 5 simulations* \\
\hline
\end{tabular}

SCO_L Difference between AWS and interpolated model elevations higher than $250 \mathrm{~m}$ in absolute value for all models

LWD Inconsistencies and period of malfunction have been evidenced from visual inspection of the time series :
QAS_U
Feb-May and Jul-Aug 2012, Feb-Jul 2015
NUK_U
Dec 2010, Jan-Aug 2011
TAS_U
Jan-Jul 2011

SCO_L Difference between AWS and interpolated model elevations higher than $250 \mathrm{~m}$ in absolute value for all models

$\begin{array}{cc}\text { SWD } & \text { QAS_U } \\ \text { NUK_U } & \text { Feb-May and Jul-Aug 2012, Nov-Dec 2013, Jan-Apr 2014 and Feb-Jul } 2015 \\ \text { NUK_L } & \text { Jan-Aug } 2011 \\ \text { TAS_U } & \text { Jan-Jun } 2011 \\ \end{array}$

\footnotetext{
* Suggesting a likely influence of local surface conditions not represented at the model resolutions
} 


\begin{tabular}{|c|c|c|c|c|c|c|c|}
\hline \multirow[b]{2}{*}{ AWS } & \multirow[b]{2}{*}{ Lat } & \multirow[b]{2}{*}{ Lon } & \multirow[b]{2}{*}{ Elev. (m) } & \multicolumn{4}{|c|}{ Difference in elevation $(\mathrm{m})$ with } \\
\hline & & & & MAR & ASR & E5 & EI \\
\hline KPC_L & 79.91 & -24.09 & 370 & 176 & 140 & 172 & 229 \\
\hline KPC_U & 79.83 & -25.17 & 870 & -15 & -71 & -83 & -65 \\
\hline SCO_L & 72.23 & -26.82 & 460 & 881 & 895 & 743 & 723 \\
\hline SCO_U & 72.39 & -27.24 & 970 & 245 & 385 & 360 & 350 \\
\hline TAS_A & 65.78 & -38.90 & 890 & -95 & -251 & -401 & 87 \\
\hline QAS_U & 61.18 & -46.82 & 900 & -42 & -292 & -55 & 233 \\
\hline QAS_A & 61.24 & -46.73 & 1000 & 20 & -167 & -139 & 159 \\
\hline NUK_U & 64.51 & -49.27 & 1120 & -51 & -104 & -16 & 22 \\
\hline NUK_N & 64.95 & -49.89 & 920 & -67 & -92 & -84 & 30 \\
\hline KAN_L & 67.10 & -49.95 & 670 & -112 & -112 & -70 & 164 \\
\hline KAN_M & 67.07 & -48.83 & 1270 & 12 & -10 & 30 & -12 \\
\hline KAN_U & 67.00 & -47.02 & 1840 & 3 & 1 & -3 & -30 \\
\hline UPE_U & 72.89 & -53.57 & 940 & 33 & -3 & -27 & -8 \\
\hline THU_L & 76.40 & -68.27 & 570 & -43 & -106 & -280 & -187 \\
\hline THU_U & 76.42 & -68.14 & 760 & -159 & -281 & -454 & -379 \\
\hline NUK_L & 64.48 & -49.54 & 530 & 299 & 216 & 298 & 537 \\
\hline QAS_L & 61.03 & -46.85 & 280 & 119 & 6 & 151 & 770 \\
\hline QAS_M & 61.10 & -46.83 & 630 & -31 & -251 & 117 & 451 \\
\hline TAS_L & 65.64 & -38.90 & 250 & 14 & -29 & 239 & 736 \\
\hline TAS_U & 65.70 & -38.87 & 570 & -142 & -275 & -83 & 410 \\
\hline UPE_L & 72.89 & -54.30 & 220 & 2 & 319 & 350 & 449 \\
\hline
\end{tabular}

Table S2. Differences in elevation (m) with AWS of the PROMICE network for the four models (MAR, ASR, E5 and E5). 


\begin{tabular}{|c|c|c|c|c|c|c|c|c|}
\hline \multirow{2}{*}{$\begin{array}{c}\text { Surface pressure }(\mathrm{hPa}) \\
\text { MAR }_{\mathrm{E} 5}\end{array}$} & \multicolumn{4}{|c|}{ Annually } & \multicolumn{4}{|c|}{ Summer } \\
\hline & $\mathrm{MB}$ & RMSE & RMSEc & $\mathrm{r}$ & MB & RMSE & RMSEc & $\mathrm{r}$ \\
\hline KPC_L & -22.62 & 22.66 & 1.47 & 0.99 & -21.63 & 21.66 & 1.01 & 0.99 \\
\hline KPC_U & 0.59 & 1.27 & 1.12 & 0.99 & 0.21 & 0.9 & 0.88 & 0.99 \\
\hline SCO_L* & -102.07 & 102.12 & 3.1 & 0.96 & -98.48 & 98.49 & 1.53 & 0.98 \\
\hline SCO_U & -28.08 & 28.11 & 1.28 & 0.99 & -27.29 & 27.31 & 0.94 & 0.99 \\
\hline TAS_A* & 11.37 & 11.97 & 3.76 & 0.96 & 11.02 & 11.05 & 0.83 & 0.99 \\
\hline QAS_U & 3.73 & 4.15 & 1.83 & 0.99 & 3.65 & 3.77 & 0.92 & 0.99 \\
\hline QAS_A* & -1.84 & 4.23 & 3.81 & 0.95 & -0.87 & 4.26 & 4.17 & 0.83 \\
\hline NUK_U & 5.2 & 5.31 & 1.03 & 1 & 4.87 & 4.94 & 0.84 & 0.99 \\
\hline NUK_N & 5.78 & 5.86 & 0.98 & 1 & 5.34 & 5.4 & 0.77 & 0.99 \\
\hline KAN_L & 12.94 & 12.98 & 0.98 & 1 & 12.27 & 12.29 & 0.76 & 0.99 \\
\hline KAN_M & -2.58 & 2.73 & 0.88 & 1 & -2.7 & 2.77 & 0.63 & 1 \\
\hline KAN_U & -0.84 & 1.2 & 0.85 & 1 & -0.9 & 1.12 & 0.67 & 0.99 \\
\hline UPE_U & -2.38 & 2.98 & 1.78 & 0.99 & -2.64 & 3.1 & 1.63 & 0.97 \\
\hline THU_L & 1.84 & 2.21 & 1.22 & 0.99 & 1.6 & 1.82 & 0.87 & 0.99 \\
\hline THU_U & 16.65 & 16.69 & 1.13 & 1 & 15.59 & 15.6 & 0.56 & 1 \\
\hline NUK_L & -34.72 & 34.78 & 2.03 & 0.98 & -33.72 & 33.75 & 1.43 & 0.98 \\
\hline QAS_L & -13.67 & 13.79 & 1.87 & 0.99 & -13.57 & 13.66 & 1.49 & 0.98 \\
\hline QAS_M & 2.42 & 2.72 & 1.22 & 0.99 & 2.39 & 2.47 & 0.61 & 1 \\
\hline TAS_L* & -0.33 & 8.35 & 8.34 & 0.84 & 0.19 & 10.07 & 10.07 & 0.45 \\
\hline TAS_U & 17.28 & 17.36 & 1.66 & 0.99 & 16.34 & 16.37 & 0.98 & 0.99 \\
\hline UPE_L & -1.69 & 2.1 & 1.25 & 0.99 & -1.8 & 2.08 & 1.03 & 0.99 \\
\hline All AWS & -8.42 & 16.17 & 1.83 & 0.98 & -8.54 & 15.75 & 1.41 & 0.96 \\
\hline 17 selected AWS & -2.87 & 10.71 & 1.35 & 0.99 & -3.18 & 10.35 & 0.97 & 0.99 \\
\hline
\end{tabular}

* Dissmissed in the study. see Table S1

Table S3. Annual and summer surface pressure (hPa) mean bias (MB). RMSE. centered RMSE (RMSEc) and correlation (r) between daily observations at PROMICE AWS and MARE5 over 2010 - 2016. 


\begin{tabular}{|c|c|c|c|c|c|c|c|c|}
\hline \multirow{2}{*}{$\begin{array}{c}\text { Surface pressure }(\mathrm{hPa}) \\
\text { MAR }_{\mathrm{EI}}\end{array}$} & \multicolumn{4}{|c|}{ Annually } & \multicolumn{4}{|c|}{ Summer } \\
\hline & $\mathrm{MB}$ & RMSE & RMSEc & $\mathrm{r}$ & MB & RMSE & RMSEc & $r$ \\
\hline KPC_L & -21.86 & 21.9 & 1.37 & 0.99 & -20.87 & 20.89 & 0.96 & 0.99 \\
\hline KPC_U & 1.35 & 1.7 & 1.03 & 0.99 & 0.99 & 1.29 & 0.82 & 0.99 \\
\hline SCO_L* & -101.47 & 101.52 & 3.11 & 0.96 & -97.79 & 97.81 & 1.56 & 0.97 \\
\hline SCO_U & -27.46 & 27.49 & 1.25 & 0.99 & -26.59 & 26.61 & 0.92 & 0.99 \\
\hline TAS_A* & 11.85 & 12.44 & 3.78 & 0.96 & 11.48 & 11.51 & 0.78 & 0.99 \\
\hline QAS_U & 4.3 & 4.71 & 1.91 & 0.99 & 4.23 & 4.34 & 0.97 & 0.99 \\
\hline QAS_A* & -1.33 & 4.06 & 3.84 & 0.95 & -0.32 & 4.12 & 4.11 & 0.84 \\
\hline NUK_U & 5.93 & 6.03 & 1.08 & 1 & 5.59 & 5.66 & 0.87 & 0.99 \\
\hline NUK_N & 6.45 & 6.52 & 1 & 1 & 6.04 & 6.09 & 0.79 & 0.99 \\
\hline KAN_L & 13.76 & 13.79 & 0.98 & 1 & 13.08 & 13.1 & 0.77 & 0.99 \\
\hline KAN_M & -1.79 & 2 & 0.9 & 1 & -1.88 & 1.98 & 0.63 & 1 \\
\hline KAN_U & -0.09 & 0.9 & 0.9 & 1 & -0.1 & 0.68 & 0.68 & 0.99 \\
\hline UPE_U & -1.28 & 2.21 & 1.8 & 0.99 & -1.56 & 2.26 & 1.64 & 0.97 \\
\hline THU_L & 3.92 & 4.09 & 1.18 & 0.99 & 3.61 & 3.71 & 0.84 & 0.99 \\
\hline THU_U & 18.73 & 18.76 & 1.13 & 1 & 17.58 & 17.59 & 0.65 & 1 \\
\hline NUK_L & -34 & 34.07 & 2.08 & 0.98 & -33.01 & 33.04 & 1.48 & 0.97 \\
\hline QAS_L & -13.09 & 13.24 & 1.98 & 0.99 & -13.01 & 13.1 & 1.54 & 0.98 \\
\hline QAS_M & 2.99 & 3.29 & 1.37 & 0.99 & 3.08 & 3.13 & 0.58 & 1 \\
\hline TAS_L* & 0.18 & 8.32 & 8.32 & 0.84 & 0.58 & 10.04 & 10.02 & 0.46 \\
\hline TAS_U & 17.76 & 17.85 & 1.74 & 0.99 & 16.75 & 16.78 & 0.99 & 0.99 \\
\hline UPE_L & -0.46 & 1.33 & 1.25 & 0.99 & -0.68 & 1.25 & 1.04 & 0.99 \\
\hline All AWS & -7.58 & 16.22 & 1.85 & 0.98 & -7.69 & 15.77 & 1.42 & 0.96 \\
\hline 17 selected AWS & -1.97 & 10.8 & 1.37 & 0.99 & -2.28 & 10.41 & 0.99 & 0.99 \\
\hline
\end{tabular}

* Dissmissed in the study. see Table S1

Table S4. Same as Table S3 but for MAR $\mathrm{EI}_{\text {. }}$ 


\begin{tabular}{|c|c|c|c|c|c|c|c|c|}
\hline \multirow{2}{*}{$\begin{array}{c}\text { Surface pressure }(\mathrm{hPa}) \\
\text { ASR }\end{array}$} & \multicolumn{4}{|c|}{ Annually } & \multicolumn{4}{|c|}{ Summer } \\
\hline & $\mathrm{MB}$ & RMSE & RMSEc & $\mathrm{r}$ & $\mathrm{MB}$ & RMSE & RMSEc & $\mathrm{r}$ \\
\hline KPC_L & -16.69 & 16.72 & 0.98 & 0.99 & -16.06 & 16.08 & 0.71 & 0.99 \\
\hline KPC_U & 8.72 & 8.77 & 0.97 & 1 & 7.92 & 7.94 & 0.64 & 1 \\
\hline SCO_L* & -102.45 & 102.5 & 3.24 & 0.95 & -98.62 & 98.63 & 1.46 & 0.98 \\
\hline SCO_U & -42.31 & 42.33 & 1.42 & 0.99 & -40.88 & 40.88 & 0.69 & 0.99 \\
\hline TAS_A* & 29.88 & 30.09 & 3.59 & 0.97 & 29.49 & 29.5 & 0.59 & 1 \\
\hline QAS_U & 33.12 & 33.16 & 1.69 & 0.99 & 32.72 & 32.73 & 0.72 & 0.99 \\
\hline QAS_A* & 19.74 & 20.09 & 3.73 & 0.95 & 20.43 & 20.83 & 4.07 & 0.84 \\
\hline NUK_U & 11.58 & 11.62 & 0.88 & 1 & 11.34 & 11.35 & 0.66 & 1 \\
\hline NUK_N & 9.58 & 9.61 & 0.71 & 1 & 9.29 & 9.31 & 0.55 & 1 \\
\hline KAN_L & 13.57 & 13.58 & 0.62 & 1 & 13.13 & 13.14 & 0.42 & 1 \\
\hline KAN_M & 0.63 & 0.83 & 0.55 & 1 & 0.61 & 0.7 & 0.34 & 1 \\
\hline KAN_U & 0.21 & 0.59 & 0.55 & 1 & 0.25 & 0.47 & 0.4 & 1 \\
\hline UPE_U & 3.08 & 3.49 & 1.63 & 0.99 & 2.75 & 3.12 & 1.47 & 0.98 \\
\hline THU_L & 11.92 & 11.98 & 1.16 & 0.99 & 11.33 & 11.36 & 0.79 & 0.99 \\
\hline THU_U & 33.51 & 33.55 & 1.51 & 0.99 & 31.82 & 31.83 & 0.65 & 1 \\
\hline NUK_L & -24.21 & 24.27 & 1.69 & 0.99 & -23.31 & 23.34 & 1.21 & 0.98 \\
\hline QAS_L & 0.07 & 1.48 & 1.47 & 0.99 & 0.06 & 1.29 & 1.29 & 0.98 \\
\hline QAS_M & 28.5 & 28.51 & 0.77 & 1 & 28.08 & 28.08 & 0.32 & 1 \\
\hline TAS_L* & 5.39 & 9.82 & 8.21 & 0.85 & 5.98 & 11.56 & 9.9 & 0.47 \\
\hline TAS_U & 33.47 & 33.49 & 1.12 & 1 & 32.38 & 32.39 & 0.6 & 1 \\
\hline UPE_L & -38.94 & 38.98 & 1.73 & 0.99 & -37.38 & 37.4 & 1.07 & 0.99 \\
\hline All AWS & -3.34 & 23.05 & 1.69 & 0.98 & -3.62 & 22.34 & 1.22 & 0.97 \\
\hline 17 selected AWS & 1.85 & 17.76 & 1.19 & 0.99 & 1.44 & 17.16 & 0.78 & 0.99 \\
\hline
\end{tabular}

* Dissmissed in the study. see Table S1

Table S5. Same as Table S3 but for ASR. 


\begin{tabular}{|c|c|c|c|c|c|c|c|c|}
\hline \multirow{2}{*}{$\begin{array}{c}\text { Surface pressure }(\mathrm{hPa}) \\
\text { ERA5 }\end{array}$} & \multicolumn{4}{|c|}{ Annually } & \multicolumn{4}{|c|}{ Summer } \\
\hline & $\mathrm{MB}$ & RMSE & RMSEc & $\mathrm{r}$ & MB & RMSE & RMSEc & $\mathrm{r}$ \\
\hline KPC_L & -20.65 & 20.67 & 0.86 & 1 & -19.81 & 19.81 & 0.45 & 1 \\
\hline KPC_U & 10.24 & 10.28 & 0.89 & 1 & 9.32 & 9.34 & 0.5 & 1 \\
\hline SCO_L* & -85.12 & 85.17 & 2.69 & 0.97 & -82 & 82.01 & 1.26 & 0.98 \\
\hline SCO_U & -39.21 & 39.23 & 1.23 & 0.99 & -38.03 & 38.04 & 0.67 & 0.99 \\
\hline TAS_A* & 48.51 & 48.65 & 3.65 & 0.97 & 47.81 & 47.82 & 0.7 & 1 \\
\hline QAS_U & 6.58 & 6.77 & 1.56 & 0.99 & 6.52 & 6.56 & 0.68 & 0.99 \\
\hline QAS_A* & 17.47 & 17.85 & 3.67 & 0.95 & 17.99 & 18.45 & 4.06 & 0.84 \\
\hline NUK_U & 2.47 & 2.57 & 0.7 & 1 & 2.21 & 2.28 & 0.58 & 1 \\
\hline NUK_N & 9.28 & 9.29 & 0.6 & 1 & 8.73 & 8.73 & 0.38 & 1 \\
\hline KAN_L & 9.16 & 9.19 & 0.68 & 1 & 8.6 & 8.61 & 0.42 & 1 \\
\hline KAN_M & -3.44 & 3.47 & 0.43 & 1 & -3.54 & 3.55 & 0.23 & 1 \\
\hline KAN_U & 0.77 & 0.86 & 0.39 & 1 & 0.68 & 0.77 & 0.35 & 1 \\
\hline UPE_U & 6.43 & 6.64 & 1.65 & 0.99 & 5.81 & 5.99 & 1.46 & 0.98 \\
\hline THU_L & 34.1 & 34.15 & 1.83 & 0.98 & 32.54 & 32.55 & 1 & 0.99 \\
\hline THU_U & 55.66 & 55.7 & 2.34 & 0.98 & 53 & 53.01 & 0.91 & 0.99 \\
\hline NUK_L & -33.18 & 33.22 & 1.64 & 0.99 & -32.26 & 32.28 & 1.18 & 0.98 \\
\hline QAS_L & -15.65 & 15.71 & 1.39 & 0.99 & -15.41 & 15.47 & 1.27 & 0.98 \\
\hline QAS_M & -13.24 & 13.25 & 0.57 & 1 & -12.82 & 12.82 & 0.26 & 1 \\
\hline TAS_L* & -25.68 & 26.97 & 8.24 & 0.85 & -24.49 & 26.39 & 9.84 & 0.47 \\
\hline TAS_U & 11.59 & 11.62 & 0.81 & 1 & 10.94 & 10.95 & 0.5 & 1 \\
\hline UPE_L & -42.54 & 42.57 & 1.71 & 0.99 & -40.97 & 40.99 & 1.06 & 0.99 \\
\hline All aws & -6.13 & 23.76 & 1.65 & 0.98 & -6.3 & 23.13 & 1.18 & 0.97 \\
\hline 17 aws & -1.83 & 18.45 & 1.17 & 0.99 & -1.96 & 18.03 & 0.74 & 0.99 \\
\hline
\end{tabular}

* Dissmissed in the study. see Table S1

Table S6. Same as Table S3 but for ERA5. 


\begin{tabular}{|c|c|c|c|c|c|c|c|c|}
\hline \multirow{2}{*}{$\begin{array}{c}\text { Surface pressure }(\mathrm{hPa}) \\
\text { EI }\end{array}$} & \multicolumn{4}{|c|}{ Annually } & \multicolumn{4}{|c|}{ Summer } \\
\hline & $\mathrm{MB}$ & RMSE & RMSEc & $\mathrm{r}$ & MB & RMSE & RMSEc & $\mathrm{r}$ \\
\hline KPC_L & -28.17 & 28.2 & 1.13 & 0.99 & -26.95 & 26.95 & 0.58 & 1 \\
\hline KPC_U & 7.87 & 7.91 & 0.81 & 1 & 7.25 & 7.27 & 0.53 & 1 \\
\hline *SCO_L & -83.6 & 83.64 & 2.69 & 0.97 & -80.39 & 80.4 & 1.22 & 0.99 \\
\hline SCO_U & -38.61 & 38.64 & 1.26 & 0.99 & -37.32 & 37.33 & 0.62 & 1 \\
\hline *TAS_A & -7.48 & 8.3 & 3.59 & 0.97 & -7.46 & 7.48 & 0.53 & 1 \\
\hline QAS_U & -25.55 & 25.61 & 1.7 & 0.99 & -25.02 & 25.03 & 0.81 & 0.99 \\
\hline *QAS_A & -15.89 & 16.31 & 3.68 & 0.95 & -14.7 & 15.26 & 4.1 & 0.84 \\
\hline NUK_U & -2.28 & 2.4 & 0.76 & 1 & -2.38 & 2.46 & 0.62 & 1 \\
\hline NUK_N & -4.09 & 4.13 & 0.58 & 1 & -4.14 & 4.17 & 0.46 & 1 \\
\hline KAN_L & -18.44 & 18.46 & 0.88 & 1 & -17.76 & 17.77 & 0.5 & 1 \\
\hline KAN_M & 1.27 & 1.35 & 0.47 & 1 & 1.09 & 1.13 & 0.29 & 1 \\
\hline KAN_U & 3.47 & 3.51 & 0.46 & 1 & 3.38 & 3.4 & 0.36 & 1 \\
\hline UPE_U & 4.47 & 4.76 & 1.62 & 0.99 & 3.93 & 4.19 & 1.43 & 0.98 \\
\hline THU_L & 22.23 & 22.28 & 1.51 & 0.99 & 21.16 & 21.18 & 0.96 & 0.99 \\
\hline THU_U & 45.93 & 45.97 & 2.02 & 0.98 & 43.67 & 43.68 & 0.84 & 0.99 \\
\hline NUK_L & -61.01 & 61.05 & 2.3 & 0.98 & -59.27 & 59.28 & 1.32 & 0.98 \\
\hline QAS_L & -87.14 & 87.17 & 2.56 & 0.98 & -85.47 & 85.49 & 1.7 & 0.97 \\
\hline QAS_M & -50.84 & 50.87 & 1.54 & 0.99 & -49.38 & 49.39 & 0.58 & 1 \\
\hline *TAS_L & -82.93 & 83.35 & 8.4 & 0.84 & -80.86 & 81.44 & 9.7 & 0.48 \\
\hline TAS_U & -45.31 & 45.32 & 1.24 & 1 & -45 & 45.01 & 0.79 & 0.99 \\
\hline UPE_L & -53.8 & 53.84 & 2.08 & 0.98 & -51.78 & 51.79 & 1.15 & 0.99 \\
\hline All AWS & -25.15 & 33.61 & 1.82 & 0.98 & -24.33 & 32.71 & 1.24 & 0.97 \\
\hline 17 selected AWS & -19.09 & 28.92 & 1.36 & 0.99 & -18.26 & 27.96 & 0.83 & 0.99 \\
\hline
\end{tabular}

* Dissmissed in the study. see Table S1

Table S7. Same as Table S3 but for ERA-Interim. 


\begin{tabular}{|c|c|c|c|c|c|c|c|c|}
\hline \multirow{2}{*}{$\begin{array}{c}\mathrm{T} 2 \mathrm{M}\left({ }^{\circ} \mathrm{C}\right) \\
\mathrm{MAR}_{\mathrm{E} 5}\end{array}$} & \multicolumn{4}{|c|}{ Annually } & \multicolumn{4}{|c|}{ Summer } \\
\hline & MB & RMSE & RMSEc & $\mathrm{r}$ & MB & RMSE & RMSEc & $\mathrm{r}$ \\
\hline KPC_L & 2.94 & 2.84 & 0.97 & 11.07 & 1.79 & 1.5 & 0.91 & 2.67 \\
\hline KPC_U & 2.56 & 2.53 & 0.98 & 11.66 & 1.27 & 1.18 & 0.94 & 3.11 \\
\hline SCO_L* & 5.43 & 2.96 & 0.95 & 9.16 & 4.23 & 2.11 & 0.71 & 1.54 \\
\hline SCO_U & 2.58 & 2.19 & 0.97 & 9.32 & 1.86 & 1.67 & 0.88 & 1.96 \\
\hline TAS_A* & 2.15 & 2.13 & 0.95 & 6.9 & 1.36 & 1.35 & 0.68 & 1.68 \\
\hline QAS_U & 2.55 & 2.52 & 0.94 & 7.3 & 1.14 & 1.06 & 0.76 & 1.53 \\
\hline QAS_A & 3 & 2.94 & 0.93 & 7.92 & 1.56 & 1.21 & 0.77 & 1.88 \\
\hline NUK_U & 1.86 & 1.86 & 0.98 & 8.62 & 1.81 & 1.26 & 0.85 & 1.85 \\
\hline NUK_N & 2.14 & 2.08 & 0.98 & 8.39 & 3.03 & 2.03 & 0.81 & 1.71 \\
\hline KAN_L & 2.12 & 2.1 & 0.98 & 9.57 & 1.87 & 0.88 & 0.89 & 1.88 \\
\hline KAN_M & 2.26 & 2.23 & 0.98 & 10.33 & 1.02 & 1.02 & 0.92 & 2.38 \\
\hline KAN_U & 2.58 & 2.43 & 0.97 & 10.66 & 1.39 & 1.39 & 0.91 & 3.33 \\
\hline UPE_U & 2.01 & 2.01 & 0.98 & 10.14 & 0.9 & 0.79 & 0.96 & 2.42 \\
\hline THU_L & 2.98 & 2.32 & 0.98 & 10.31 & 1.55 & 1.13 & 0.93 & 2.77 \\
\hline THU_U & 2.41 & 2.16 & 0.98 & 10.41 & 1.16 & 0.98 & 0.94 & 2.91 \\
\hline NUK_L* & 3.51 & 2.9 & 0.96 & 8.06 & 1.92 & 1.83 & 0.71 & 1.96 \\
\hline QAS_L & 2.1 & 2.03 & 0.97 & 6.28 & 2.27 & 1.38 & 0.77 & 1.5 \\
\hline QAS_M & 1.4 & 1.37 & 0.98 & 7.16 & 1.22 & 0.58 & 0.88 & 0.93 \\
\hline TAS_L* & 2.53 & 2.49 & 0.93 & 5.35 & 3.41 & 2.11 & 0.59 & 1.34 \\
\hline TAS_U & 2.31 & 2.01 & 0.95 & 6.07 & 2.89 & 1.37 & 0.79 & 1.44 \\
\hline UPE_L & 2.75 & 2.68 & 0.97 & 9.89 & 2.45 & 1.49 & 0.82 & 2.43 \\
\hline All AWS & -0.39 & 2.65 & 2.36 & 0.97 & 0.3 & 1.93 & 1.37 & 0.83 \\
\hline 17 selected AWS & -0.04 & 2.41 & 2.28 & 0.97 & 0.44 & 1.69 & 1.24 & 0.87 \\
\hline
\end{tabular}

* Dissmissed in the study. see Table $\mathrm{S} 1$

Table S8. Annual and summer near-surface temperature (T2M, $\left.{ }^{\circ} \mathrm{C}\right)$ mean bias (MB). RMSE. centered RMSE (RMSEc) and correlation (r) between daily observations at PROMICE AWS and MARE5 over 2010 - 2016. 


\begin{tabular}{|c|c|c|c|c|c|c|c|c|}
\hline \multirow{2}{*}{$\begin{array}{c}\mathrm{T} 2 \mathrm{M}\left({ }^{\circ} \mathrm{C}\right) \\
\mathrm{MAR}_{\mathrm{EI}}\end{array}$} & \multicolumn{4}{|c|}{ Annually } & \multicolumn{4}{|c|}{ Summer } \\
\hline & $\mathrm{MB}$ & RMSE & RMSEc & $\mathrm{r}$ & $\mathrm{MB}$ & RMSE & RMSEc & $\mathrm{r}$ \\
\hline KPC_L & 2.7 & 2.65 & 0.97 & 11.07 & 1.55 & 1.46 & 0.92 & 2.67 \\
\hline KPC_U & 2.43 & 2.33 & 0.98 & 11.66 & 1.1 & 1.1 & 0.94 & 3.11 \\
\hline SCO_L* & 5.4 & 2.92 & 0.95 & 9.16 & 4 & 2.06 & 0.71 & 1.54 \\
\hline SCO_U & 2.56 & 2.18 & 0.98 & 9.32 & 1.71 & 1.61 & 0.88 & 1.96 \\
\hline TAS_A* & 2.21 & 2.19 & 0.95 & 6.9 & 1.33 & 1.3 & 0.69 & 1.68 \\
\hline QAS_U & 2.56 & 2.52 & 0.94 & 7.3 & 1.16 & 1.03 & 0.77 & 1.53 \\
\hline QAS_A & 3.07 & 3.03 & 0.93 & 7.92 & 1.51 & 1.23 & 0.75 & 1.88 \\
\hline NUK_U & 1.9 & 1.9 & 0.98 & 8.62 & 1.93 & 1.23 & 0.85 & 1.85 \\
\hline NUK_N & 2.21 & 2.11 & 0.98 & 8.39 & 3.23 & 1.96 & 0.81 & 1.71 \\
\hline KAN_L & 2.12 & 2.08 & 0.98 & 9.57 & 1.95 & 0.89 & 0.89 & 1.88 \\
\hline KAN_M & 2.16 & 2.13 & 0.98 & 10.33 & 0.98 & 0.97 & 0.93 & 2.38 \\
\hline KAN_U & 2.55 & 2.4 & 0.97 & 10.66 & 1.42 & 1.42 & 0.91 & 3.33 \\
\hline UPE_U & 2.01 & 2.01 & 0.98 & 10.14 & 0.89 & 0.82 & 0.95 & 2.42 \\
\hline THU_L & 3.09 & 2.56 & 0.98 & 10.31 & 1.24 & 1.24 & 0.92 & 2.77 \\
\hline THU_U & 2.52 & 2.33 & 0.98 & 10.41 & 1.05 & 1.03 & 0.94 & 2.91 \\
\hline NUK_L* & 3.48 & 2.96 & 0.96 & 8.06 & 2 & 1.84 & 0.7 & 1.96 \\
\hline QAS_L & 2.15 & 2.1 & 0.96 & 6.28 & 2.37 & 1.4 & 0.76 & 1.5 \\
\hline QAS_M & 1.41 & 1.36 & 0.98 & 7.16 & 1.27 & 0.58 & 0.86 & 0.93 \\
\hline TAS_L* & 2.65 & 2.59 & 0.93 & 5.35 & 3.65 & 1.96 & 0.64 & 1.34 \\
\hline TAS_U & 2.44 & 2.09 & 0.95 & 6.07 & 3.24 & 1.34 & 0.79 & 1.44 \\
\hline UPE_L & 2.81 & 2.8 & 0.97 & 9.89 & 2.5 & 1.52 & 0.82 & 2.43 \\
\hline All AWS & -0.34 & 2.66 & 2.38 & 0.97 & 0.58 & 1.93 & 1.36 & 0.84 \\
\hline 17 selected AWS & 0.01 & 2.42 & 2.29 & 0.97 & 0.72 & 1.68 & 1.24 & 0.87 \\
\hline
\end{tabular}

* Dissmissed in the study. see Table $\mathrm{S} 1$

Table S9. Same as Table S8 but for MAR $\mathrm{EI}_{\text {. }}$ 


\begin{tabular}{|c|c|c|c|c|c|c|c|c|}
\hline \multirow{2}{*}{$\begin{array}{c}\mathrm{T} 2 \mathrm{M}\left({ }^{\circ} \mathrm{C}\right) \\
\text { ASR }\end{array}$} & \multicolumn{4}{|c|}{ Annually } & \multicolumn{4}{|c|}{ Summer } \\
\hline & MB & RMSE & RMSEc & $\mathrm{r}$ & MB & RMSE & RMSEc & $\mathrm{r}$ \\
\hline KPC_L & -2.74 & 3.92 & 2.81 & 0.97 & -2.06 & 2.66 & 1.69 & 0.87 \\
\hline KPC_U & -0.27 & 1.98 & 1.96 & 0.99 & -0.78 & 1.35 & 1.1 & 0.94 \\
\hline SCO_L* & -5.36 & 6.04 & 2.77 & 0.96 & -4.96 & 5.26 & 1.75 & 0.71 \\
\hline SCO_U & -3.66 & 4.12 & 1.88 & 0.98 & -3.57 & 3.77 & 1.23 & 0.9 \\
\hline TAS_A* & 1.23 & 2.22 & 1.85 & 0.97 & 0.14 & 1.32 & 1.31 & 0.71 \\
\hline QAS_U & 2.06 & 3.31 & 2.59 & 0.94 & 1.66 & 2.11 & 1.3 & 0.71 \\
\hline QAS_A & 0.82 & 3.2 & 3.09 & 0.92 & -0.29 & 1.34 & 1.3 & 0.73 \\
\hline NUK_U & -0.37 & 1.86 & 1.82 & 0.98 & 0.54 & 1.27 & 1.15 & 0.88 \\
\hline NUK_N & 0.9 & 2.07 & 1.86 & 0.99 & 2.84 & 3.12 & 1.29 & 0.92 \\
\hline KAN_L & 0.49 & 1.95 & 1.89 & 0.99 & 2.09 & 2.22 & 0.75 & 0.95 \\
\hline KAN_M & -0.51 & 2.09 & 2.03 & 0.98 & -0.7 & 1.49 & 1.32 & 0.89 \\
\hline KAN_U & -1.28 & 2.67 & 2.34 & 0.98 & -1.64 & 2.28 & 1.59 & 0.9 \\
\hline UPE_U & -0.23 & 1.83 & 1.82 & 0.98 & -0.16 & 0.95 & 0.94 & 0.95 \\
\hline THU_L & -1.73 & 3.01 & 2.46 & 0.98 & -0.94 & 1.97 & 1.74 & 0.78 \\
\hline THU_U & -0.67 & 2.51 & 2.42 & 0.98 & -0.14 & 1.77 & 1.77 & 0.79 \\
\hline NUK_L* & -1.63 & 3.66 & 3.28 & 0.96 & 1.38 & 2.2 & 1.71 & 0.74 \\
\hline QAS_L & 0.13 & 1.68 & 1.67 & 0.98 & 2.09 & 2.28 & 0.91 & 0.88 \\
\hline QAS_M & 1.47 & 1.85 & 1.13 & 0.99 & 1.79 & 1.93 & 0.73 & 0.92 \\
\hline TAS_* & 0.13 & 1.48 & 1.47 & 0.97 & 0.85 & 1.5 & 1.23 & 0.72 \\
\hline TAS_U & 1.51 & 2.03 & 1.36 & 0.97 & 1.39 & 1.67 & 0.94 & 0.83 \\
\hline UPE_L & -1.52 & 3.34 & 2.98 & 0.96 & -0.74 & 1.58 & 1.39 & 0.88 \\
\hline All AWS & -0.75 & 2.76 & 2.2 & 0.97 & -0.23 & 2.15 & 1.32 & 0.84 \\
\hline 17 selected AWS & -0.47 & 2.54 & 2.14 & 0.98 & -0.07 & 1.98 & 1.26 & 0.87 \\
\hline
\end{tabular}

* Dissmissed in the study. see Table S1

Table S10. Same as Table S8 but for ASR. 


\begin{tabular}{|c|c|c|c|c|c|c|c|c|}
\hline \multirow{2}{*}{$\begin{array}{c}\mathrm{T} 2 \mathrm{M}\left({ }^{\circ} \mathrm{C}\right) \\
\text { E5 }\end{array}$} & \multicolumn{4}{|c|}{ Annually } & \multicolumn{4}{|c|}{ Summer } \\
\hline & MB & RMSE & RMSEc & $\mathrm{r}$ & MB & RMSE & RMSEc & $\mathrm{r}$ \\
\hline KPC_L & -2.15 & 2.72 & 1.66 & 0.99 & -2.46 & 2.79 & 1.3 & 0.92 \\
\hline KPC_U & 1.62 & 2.72 & 2.19 & 0.98 & 0.24 & 1.35 & 1.33 & 0.92 \\
\hline SCO_L* & -7.96 & 8.59 & 3.21 & 0.95 & -5.64 & 6.1 & 2.31 & 0.65 \\
\hline SCO_U & -6.27 & 6.83 & 2.72 & 0.97 & -4.96 & 5.31 & 1.87 & 0.81 \\
\hline TAS_A* & 2.75 & 3.58 & 2.28 & 0.95 & 1 & 1.85 & 1.56 & 0.55 \\
\hline QAS_U & 1.85 & 3.32 & 2.75 & 0.93 & 1.27 & 1.91 & 1.43 & 0.72 \\
\hline QAS_A & 1.94 & 3.76 & 3.23 & 0.91 & 0.28 & 1.13 & 1.1 & 0.85 \\
\hline NUK_U & -0.05 & 2.17 & 2.17 & 0.97 & -1.54 & 2.34 & 1.76 & 0.75 \\
\hline NUK_N & 1.48 & 2.37 & 1.85 & 0.98 & 3.15 & 3.56 & 1.66 & 0.88 \\
\hline KAN_L & 1.63 & 2.96 & 2.47 & 0.98 & 4.43 & 4.64 & 1.39 & 0.91 \\
\hline KAN_M & 1.49 & 2.97 & 2.56 & 0.98 & -1.22 & 1.88 & 1.43 & 0.86 \\
\hline KAN_U & 1.37 & 3.12 & 2.8 & 0.98 & -1.75 & 2.44 & 1.71 & 0.87 \\
\hline UPE_U & 0.27 & 1.77 & 1.75 & 0.99 & -0.54 & 1.33 & 1.21 & 0.91 \\
\hline THU_L & -2.33 & 5.36 & 4.82 & 0.96 & 2.29 & 2.97 & 1.9 & 0.85 \\
\hline THU_U & -1.42 & 5.03 & 4.83 & 0.97 & 3.06 & 3.54 & 1.77 & 0.87 \\
\hline NUK_L* & -2.33 & 3.27 & 2.29 & 0.96 & -2.02 & 2.81 & 1.95 & 0.62 \\
\hline QAS_L & -0.07 & 1.73 & 1.73 & 0.98 & 1.76 & 2.07 & 1.09 & 0.88 \\
\hline QAS_M & 0.37 & 2.02 & 1.99 & 0.96 & 0.03 & 1.16 & 1.16 & 0.87 \\
\hline TAS_L* & -0.68 & 1.63 & 1.49 & 0.97 & 0.11 & 1.33 & 1.33 & 0.67 \\
\hline TAS_U & 1.23 & 1.85 & 1.38 & 0.97 & 1.16 & 1.62 & 1.14 & 0.74 \\
\hline UPE_L & -1.91 & 3.11 & 2.45 & 0.97 & -1.42 & 2 & 1.42 & 0.87 \\
\hline All AWS & -0.66 & 3.45 & 2.51 & 0.97 & -0.28 & 2.74 & 1.56 & 0.81 \\
\hline 17 selected AWS & -0.11 & 3.18 & 2.55 & 0.97 & 0.19 & 2.6 & 1.49 & 0.85 \\
\hline
\end{tabular}

* Dissmissed in the study. see Table S1

Table S11. Same as Table S8 but for ERA5. 


\begin{tabular}{|c|c|c|c|c|c|c|c|c|}
\hline \multirow{2}{*}{$\begin{array}{c}\mathrm{T} 2 \mathrm{M}\left({ }^{\circ} \mathrm{C}\right) \\
\mathrm{EI}\end{array}$} & \multicolumn{4}{|c|}{ Annually } & \multicolumn{4}{|c|}{ Summer } \\
\hline & MB & RMSE & RMSEc & $\mathrm{r}$ & MB & RMSE & RMSEc & $\mathrm{r}$ \\
\hline KPC_L & -3.44 & 4.48 & 2.87 & 0.98 & -2.09 & 2.49 & 1.36 & 0.88 \\
\hline KPC_U & 1.27 & 2.56 & 2.22 & 0.98 & 0.75 & 1.47 & 1.27 & 0.91 \\
\hline SCO_L* & -6.23 & 7 & 3.19 & 0.96 & -3.38 & 4.05 & 2.24 & 0.77 \\
\hline SCO_U & -5.43 & 6.26 & 3.12 & 0.97 & -2.96 & 3.58 & 2.01 & 0.83 \\
\hline TAS_A* & -1.85 & 3.15 & 2.55 & 0.95 & -0.9 & 1.85 & 1.62 & 0.62 \\
\hline QAS_U & -1.24 & 4.1 & 3.9 & 0.93 & 0.75 & 1.63 & 1.44 & 0.7 \\
\hline QAS_A & -1.81 & 4.55 & 4.17 & 0.91 & -0.41 & 1.11 & 1.03 & 0.88 \\
\hline NUK_U & -0.13 & 2.41 & 2.4 & 0.97 & 1.19 & 1.76 & 1.3 & 0.8 \\
\hline NUK_N & 0.33 & 2.21 & 2.18 & 0.98 & 1.8 & 2.19 & 1.25 & 0.83 \\
\hline KAN_L & -0.6 & 2.56 & 2.48 & 0.98 & 1.17 & 1.63 & 1.14 & 0.88 \\
\hline KAN_M & 1.72 & 2.65 & 2.01 & 0.98 & 1.3 & 1.76 & 1.19 & 0.88 \\
\hline KAN_U & 2.06 & 2.95 & 2.12 & 0.98 & 0.58 & 1.63 & 1.52 & 0.89 \\
\hline UPE_U & -0.32 & 2.75 & 2.73 & 0.97 & 0.97 & 1.6 & 1.28 & 0.92 \\
\hline THU_L & -0.4 & 3.59 & 3.57 & 0.96 & 1.07 & 1.86 & 1.52 & 0.85 \\
\hline THU_U & 0.73 & 3.67 & 3.6 & 0.96 & 2.11 & 2.62 & 1.55 & 0.87 \\
\hline NUK_L* & -3.56 & 4.65 & 3 & 0.96 & -1.3 & 2.17 & 1.73 & 0.64 \\
\hline QAS_L & -4.81 & 5.92 & 3.44 & 0.96 & -1.4 & 2.04 & 1.49 & 0.68 \\
\hline QAS_M & -4.23 & 5.38 & 3.31 & 0.97 & -1.49 & 1.72 & 0.84 & 0.92 \\
\hline TAS_L* & -5.39 & 6.39 & 3.43 & 0.95 & -1.79 & 2.43 & 1.65 & 0.63 \\
\hline TAS_U & -3.57 & 4.61 & 2.92 & 0.96 & -0.6 & 1.64 & 1.53 & 0.66 \\
\hline UPE_L & -2.58 & 3.79 & 2.77 & 0.96 & -1.23 & 1.86 & 1.39 & 0.87 \\
\hline All AWS & -1.77 & 4.03 & 2.9 & 0.96 & -0.22 & 2.11 & 1.5 & 0.8 \\
\hline 17 selected AWS & -1.08 & 3.65 & 2.85 & 0.97 & 0.22 & 1.95 & 1.41 & 0.83 \\
\hline
\end{tabular}

* Dissmissed in the study. see Table S1

Table S12. Same as Table S8 but for ERA-Interim. 


\begin{tabular}{|c|c|c|c|c|c|c|c|c|}
\hline \multirow{2}{*}{$\begin{array}{c}\mathrm{W} 10 \mathrm{M}\left(\mathrm{ms}^{-1}\right) \\
\text { MAR }_{\mathrm{E} 5}\end{array}$} & \multicolumn{4}{|c|}{ Annually } & \multicolumn{4}{|c|}{ Summer } \\
\hline & MB & RMSE & RMSEc & $\mathrm{r}$ & MB & RMSE & RMSEc & $\mathrm{r}$ \\
\hline KPC_L & 0.4 & 1.94 & 1.9 & 0.73 & -0.59 & 1.5 & 1.38 & 0.7 \\
\hline KPC_U & 0.96 & 1.61 & 1.29 & 0.83 & 0.39 & 1.12 & 1.05 & 0.79 \\
\hline SCO_L* & 1.7 & 2.32 & 1.57 & 0.54 & 1.15 & 1.67 & 1.21 & 0.53 \\
\hline SCO_U* & -0.34 & 1.64 & 1.61 & 0.45 & -0.22 & 1.25 & 1.23 & 0.41 \\
\hline TAS_A & 1.98 & 3.26 & 2.6 & 0.83 & 1.74 & 2.31 & 1.52 & 0.78 \\
\hline QAS_U & 2.23 & 3 & 2.01 & 0.85 & 1.84 & 2.29 & 1.36 & 0.71 \\
\hline QAS_A* & 1.31 & 2.64 & 2.29 & 0.76 & 0.71 & 1.42 & 1.22 & 0.73 \\
\hline NUK_U & 1.49 & 2.34 & 1.81 & 0.84 & 1.24 & 1.75 & 1.23 & 0.73 \\
\hline NUK_N & 1.29 & 1.92 & 1.42 & 0.88 & 0.87 & 1.28 & 0.94 & 0.83 \\
\hline KAN_L & 1.88 & 2.29 & 1.31 & 0.85 & 2.2 & 2.45 & 1.07 & 0.79 \\
\hline KAN_M & 0.79 & 1.58 & 1.37 & 0.9 & 1.04 & 1.52 & 1.11 & 0.78 \\
\hline KAN_U & 0.31 & 1.36 & 1.32 & 0.93 & -0.05 & 0.96 & 0.96 & 0.89 \\
\hline UPE_U & 1.33 & 2.38 & 1.98 & 0.8 & 1.59 & 2.32 & 1.69 & 0.72 \\
\hline THU_L & 1.89 & 2.99 & 2.32 & 0.79 & 1.48 & 2.52 & 2.04 & 0.85 \\
\hline THU_U & 1.38 & 2.57 & 2.17 & 0.79 & 0.93 & 2.06 & 1.83 & 0.87 \\
\hline NUK_L* & 2.69 & 3.4 & 2.08 & 0.77 & 1.71 & 2.18 & 1.35 & 0.64 \\
\hline QAS_L & 1.84 & 2.73 & 2.02 & 0.83 & 0.72 & 1.53 & 1.35 & 0.68 \\
\hline QAS_M & 1.18 & 1.9 & 1.49 & 0.92 & 0.34 & 0.77 & 0.69 & 0.75 \\
\hline TAS_L & 1.94 & 3.53 & 2.95 & 0.79 & 0.5 & 1.74 & 1.67 & 0.56 \\
\hline TAS_U & 1.94 & 3.31 & 2.68 & 0.81 & 1.2 & 1.99 & 1.58 & 0.68 \\
\hline UPE_L & 1.62 & 2.58 & 2 & 0.74 & 1.48 & 2.08 & 1.46 & 0.77 \\
\hline All AWS & 1.4 & 2.4 & 1.86 & 0.78 & 1.01 & 1.8 & 1.35 & 0.72 \\
\hline 17 selected AWS & 1.42 & 2.39 & 1.87 & 0.83 & 1.05 & 1.83 & 1.37 & 0.76 \\
\hline
\end{tabular}

* Dissmissed in the study. see Table S1

Table S13. Annual and summer 10-m wind speed (W10M) mean bias (MB). RMSE. centered RMSE (RMSEc) and correlation (r) between

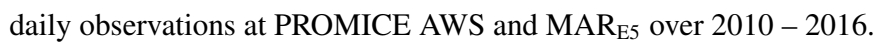




\begin{tabular}{|c|c|c|c|c|c|c|c|c|}
\hline \multirow{2}{*}{$\begin{array}{c}\mathrm{UV} 2\left(\mathrm{~ms}^{-1}\right) \\
\text { MAR }_{\mathrm{E} 5}\end{array}$} & \multicolumn{4}{|c|}{ Annually } & \multicolumn{4}{|c|}{ Summer } \\
\hline & MB & RMSE & RMSEc & $\mathrm{r}$ & MB & RMSE & RMSEc & $\mathrm{r}$ \\
\hline KPC_L & -1.04 & 2.11 & 1.83 & 0.75 & -1.73 & 2.24 & 1.42 & 0.69 \\
\hline KPC_U & -0.41 & 1.32 & 1.25 & 0.83 & -0.67 & 1.24 & 1.04 & 0.78 \\
\hline SCO_L* & 0.4 & 1.62 & 1.57 & 0.5 & 0.11 & 1.25 & 1.24 & 0.5 \\
\hline SCO_U* & -1.58 & 2.23 & 1.58 & 0.42 & -1.3 & 1.78 & 1.22 & 0.41 \\
\hline TAS_A & 0.25 & 2.64 & 2.63 & 0.82 & 0.38 & 1.6 & 1.55 & 0.76 \\
\hline QAS_U & 0.41 & 2.01 & 1.96 & 0.83 & 0.38 & 1.38 & 1.32 & 0.7 \\
\hline QAS_A* & -0.47 & 2.25 & 2.2 & 0.75 & -0.67 & 1.38 & 1.21 & 0.73 \\
\hline NUK_U & -0.02 & 1.74 & 1.74 & 0.84 & -0.11 & 1.23 & 1.23 & 0.72 \\
\hline NUK_N & -0.18 & 1.41 & 1.4 & 0.87 & -0.33 & 1.05 & 1 & 0.8 \\
\hline KAN_L & 0.55 & 1.46 & 1.35 & 0.83 & 1.05 & 1.51 & 1.08 & 0.77 \\
\hline KAN_M & -0.53 & 1.51 & 1.42 & 0.89 & 0 & 1.11 & 1.11 & 0.77 \\
\hline KAN_U & -0.98 & 1.67 & 1.35 & 0.92 & -1.02 & 1.43 & 1.01 & 0.88 \\
\hline UPE_U & -0.04 & 1.91 & 1.91 & 0.8 & 0.39 & 1.66 & 1.61 & 0.72 \\
\hline THU_L & 0.38 & 2.38 & 2.35 & 0.78 & 0.13 & 2.17 & 2.16 & 0.83 \\
\hline THU_U & -0.08 & 2.21 & 2.21 & 0.78 & -0.37 & 1.99 & 1.95 & 0.85 \\
\hline NUK_L* & 1.2 & 2.3 & 1.96 & 0.76 & 0.34 & 1.36 & 1.31 & 0.64 \\
\hline QAS_L & 0.04 & 1.98 & 1.98 & 0.81 & -0.79 & 1.56 & 1.34 & 0.67 \\
\hline QAS_M & -0.61 & 1.67 & 1.55 & 0.89 & -1.04 & 1.19 & 0.59 & 0.79 \\
\hline TAS_L & 0.37 & 3.01 & 2.99 & 0.76 & -0.69 & 1.8 & 1.66 & 0.52 \\
\hline TAS_U & 0.31 & 2.74 & 2.72 & 0.78 & -0.1 & 1.55 & 1.55 & 0.66 \\
\hline UPE_L & 0.32 & 1.97 & 1.94 & 0.73 & 0.36 & 1.47 & 1.43 & 0.76 \\
\hline All AWS & -0.06 & 1.96 & 1.84 & 0.77 & -0.2 & 1.53 & 1.36 & 0.71 \\
\hline 17 selected AWS & -0.06 & 1.93 & 1.86 & 0.82 & -0.17 & 1.55 & 1.38 & 0.75 \\
\hline
\end{tabular}

* Dissmissed in the study. see Table S1

Table S14. Same as Table S13 but for AWS height wind speed (UV2). 


\begin{tabular}{|c|c|c|c|c|c|c|c|c|}
\hline \multirow{2}{*}{$\begin{array}{c}\text { W10M }\left(\mathrm{ms}^{-1}\right) \\
\text { MAR }_{\mathrm{EI}}\end{array}$} & \multicolumn{4}{|c|}{ Annually } & \multicolumn{4}{|c|}{ Summer } \\
\hline & MB & RMSE & RMSEc & $\mathrm{r}$ & MB & RMSE & RMSEc & $\mathrm{r}$ \\
\hline KPC_L & 0.54 & 1.92 & 1.84 & 0.75 & -0.44 & 1.46 & 1.39 & 0.7 \\
\hline KPC_U & 1.09 & 1.69 & 1.28 & 0.84 & 0.56 & 1.19 & 1.05 & 0.79 \\
\hline SCO_L* & 1.77 & 2.39 & 1.6 & 0.54 & 1.23 & 1.72 & 1.21 & 0.53 \\
\hline SCO_U* & -0.33 & 1.63 & 1.6 & 0.47 & -0.2 & 1.21 & 1.19 & 0.45 \\
\hline TAS_A & 2.02 & 3.24 & 2.53 & 0.84 & 1.92 & 2.45 & 1.52 & 0.79 \\
\hline QAS_U & 2.32 & 3.12 & 2.09 & 0.84 & 2 & 2.44 & 1.4 & 0.7 \\
\hline QAS_A* & 1.43 & 2.75 & 2.34 & 0.75 & 0.89 & 1.57 & 1.29 & 0.72 \\
\hline NUK_U & 1.55 & 2.41 & 1.84 & 0.83 & 1.37 & 1.9 & 1.32 & 0.7 \\
\hline NUK_N & 1.32 & 1.91 & 1.38 & 0.88 & 0.94 & 1.33 & 0.95 & 0.81 \\
\hline KAN_L & 1.85 & 2.28 & 1.33 & 0.85 & 2.21 & 2.45 & 1.06 & 0.79 \\
\hline KAN_M & 0.74 & 1.6 & 1.42 & 0.89 & 1.02 & 1.53 & 1.14 & 0.76 \\
\hline KAN_U & 0.19 & 1.33 & 1.31 & 0.92 & -0.14 & 0.93 & 0.92 & 0.9 \\
\hline UPE_U & 1.23 & 2.26 & 1.89 & 0.81 & 1.56 & 2.19 & 1.54 & 0.76 \\
\hline THU_L & 0.93 & 2.4 & 2.21 & 0.81 & 0.41 & 1.83 & 1.78 & 0.89 \\
\hline THU_U & 0.36 & 2.07 & 2.04 & 0.81 & -0.11 & 1.68 & 1.67 & 0.89 \\
\hline NUK_L* & 2.75 & 3.46 & 2.11 & 0.76 & 1.81 & 2.25 & 1.33 & 0.66 \\
\hline QAS_L & 1.88 & 2.8 & 2.08 & 0.82 & 0.78 & 1.57 & 1.37 & 0.67 \\
\hline QAS_M & 1.28 & 1.99 & 1.53 & 0.92 & 0.49 & 0.9 & 0.75 & 0.71 \\
\hline TAS_L & 1.9 & 3.44 & 2.87 & 0.8 & 0.54 & 1.77 & 1.68 & 0.56 \\
\hline TAS_U & 1.95 & 3.24 & 2.59 & 0.82 & 1.26 & 2.02 & 1.58 & 0.68 \\
\hline UPE_L & 1.5 & 2.47 & 1.96 & 0.74 & 1.42 & 1.98 & 1.38 & 0.78 \\
\hline All AWS & 1.32 & 2.35 & 1.84 & 0.79 & 0.96 & 1.77 & 1.32 & 0.73 \\
\hline 17 selected AWS & 1.31 & 2.32 & 1.85 & 0.83 & 0.96 & 1.78 & 1.34 & 0.77 \\
\hline
\end{tabular}

* Dissmissed in the study. see Table S1

Table S15. Same as Table S13 but for MAR $\mathrm{EI}_{\text {. }}$ 


\begin{tabular}{|c|c|c|c|c|c|c|c|c|}
\hline \multirow{2}{*}{$\begin{array}{c}\mathrm{UV} 2\left(\mathrm{~ms}^{-1}\right) \\
\text { MAR }_{\mathrm{EI}}\end{array}$} & \multicolumn{4}{|c|}{ Annually } & \multicolumn{4}{|c|}{ Summer } \\
\hline & $\mathrm{MB}$ & RMSE & RMSEc & $\mathrm{r}$ & MB & RMSE & RMSEc & $\mathrm{r}$ \\
\hline KPC_L & -0.9 & 1.99 & 1.77 & 0.77 & -1.57 & 2.12 & 1.42 & 0.69 \\
\hline KPC_U & -0.27 & 1.28 & 1.25 & 0.84 & -0.5 & 1.15 & 1.04 & 0.79 \\
\hline SCO_L* & 0.48 & 1.67 & 1.6 & 0.51 & 0.2 & 1.26 & 1.24 & 0.5 \\
\hline SCO_U* & -1.56 & 2.21 & 1.57 & 0.44 & -1.25 & 1.71 & 1.17 & 0.45 \\
\hline TAS_A & 0.28 & 2.59 & 2.58 & 0.83 & 0.54 & 1.65 & 1.56 & 0.76 \\
\hline QAS_U & 0.52 & 2.09 & 2.02 & 0.83 & 0.53 & 1.45 & 1.34 & 0.69 \\
\hline QAS_A* & -0.36 & 2.3 & 2.27 & 0.73 & -0.49 & 1.36 & 1.27 & 0.71 \\
\hline NUK_U & 0.04 & 1.79 & 1.79 & 0.83 & -0.01 & 1.31 & 1.31 & 0.69 \\
\hline NUK_N & -0.14 & 1.39 & 1.38 & 0.87 & -0.28 & 1.08 & 1.04 & 0.78 \\
\hline KAN_L & 0.52 & 1.46 & 1.37 & 0.83 & 1.05 & 1.5 & 1.07 & 0.77 \\
\hline KAN_M & -0.58 & 1.57 & 1.46 & 0.88 & -0.03 & 1.16 & 1.16 & 0.75 \\
\hline KAN_U & -1.1 & 1.74 & 1.35 & 0.92 & -1.11 & 1.49 & 0.99 & 0.88 \\
\hline UPE_U & -0.15 & 1.83 & 1.82 & 0.81 & 0.34 & 1.5 & 1.46 & 0.76 \\
\hline THU_L & -0.59 & 2.28 & 2.2 & 0.81 & -0.94 & 2.09 & 1.87 & 0.88 \\
\hline THU_U & -1.1 & 2.33 & 2.05 & 0.81 & -1.44 & 2.26 & 1.74 & 0.89 \\
\hline NUK_L* & 1.26 & 2.36 & 1.99 & 0.76 & 0.43 & 1.36 & 1.29 & 0.66 \\
\hline QAS_L & 0.11 & 2.03 & 2.03 & 0.8 & -0.71 & 1.51 & 1.34 & 0.67 \\
\hline QAS_M & -0.51 & 1.67 & 1.59 & 0.89 & -0.88 & 1.14 & 0.71 & 0.72 \\
\hline TAS_L & 0.34 & 2.95 & 2.93 & 0.77 & -0.7 & 1.84 & 1.7 & 0.51 \\
\hline TAS_U & 0.3 & 2.66 & 2.65 & 0.8 & -0.05 & 1.55 & 1.55 & 0.66 \\
\hline UPE_L & 0.21 & 1.91 & 1.9 & 0.74 & 0.32 & 1.38 & 1.34 & 0.78 \\
\hline All AWS & -0.13 & 1.96 & 1.82 & 0.78 & -0.26 & 1.53 & 1.32 & 0.72 \\
\hline 17 selected AWS & -0.17 & 1.93 & 1.84 & 0.82 & -0.26 & 1.55 & 1.34 & 0.75 \\
\hline
\end{tabular}

* Dissmissed in the study. see Table S1

Table S16. Same as Table S14 but for MAR $\mathrm{EI}_{\text {. }}$ 


\begin{tabular}{|c|c|c|c|c|c|c|c|c|}
\hline \multirow{2}{*}{$\begin{array}{c}\mathrm{W} 10 \mathrm{M}\left(\mathrm{ms}^{-1}\right) \\
\text { ASR }\end{array}$} & \multicolumn{4}{|c|}{ Annually } & \multicolumn{4}{|c|}{ Summer } \\
\hline & MB & RMSE & RMSEc & $\mathrm{r}$ & MB & RMSE & RMSEc & $\mathrm{r}$ \\
\hline KPC_L & 0.05 & 1.87 & 1.87 & 0.78 & -1.34 & 2.04 & 1.54 & 0.7 \\
\hline KPC_U & 1.86 & 2.39 & 1.51 & 0.84 & 0.96 & 1.53 & 1.19 & 0.81 \\
\hline SCO_L* & 2.73 & 3.4 & 2.02 & 0.54 & 2.18 & 2.6 & 1.41 & 0.58 \\
\hline SCO_U* & 1.45 & 2.37 & 1.87 & 0.56 & 1.45 & 2.04 & 1.43 & 0.53 \\
\hline TAS_A & 2.1 & 3.23 & 2.44 & 0.86 & 3.09 & 3.48 & 1.61 & 0.74 \\
\hline QAS_U & 2.22 & 3.04 & 2.07 & 0.8 & 2.35 & 2.72 & 1.38 & 0.68 \\
\hline QAS_A* & 1.67 & 2.87 & 2.33 & 0.73 & 1.65 & 2.12 & 1.34 & 0.67 \\
\hline NUK_U & 2.79 & 3.57 & 2.23 & 0.84 & 2.07 & 2.48 & 1.38 & 0.75 \\
\hline NUK_N & 1.36 & 2.2 & 1.73 & 0.88 & 0.56 & 1.12 & 0.97 & 0.84 \\
\hline KAN_L & 1.65 & 2.22 & 1.49 & 0.82 & 1.93 & 2.3 & 1.25 & 0.76 \\
\hline KAN_M & 1.98 & 2.59 & 1.67 & 0.88 & 1.96 & 2.4 & 1.38 & 0.76 \\
\hline KAN_U & 0.84 & 1.62 & 1.39 & 0.93 & 0.29 & 1.13 & 1.09 & 0.89 \\
\hline UPE_U & 2.51 & 3.06 & 1.76 & 0.89 & 2.43 & 2.82 & 1.44 & 0.85 \\
\hline THU_L & -1.38 & 2.76 & 2.39 & 0.77 & -2.02 & 2.83 & 1.98 & 0.86 \\
\hline THU_U & -1.45 & 2.79 & 2.39 & 0.74 & -2.16 & 3.01 & 2.09 & 0.83 \\
\hline NUK_L* & 2.79 & 3.69 & 2.41 & 0.78 & 1.31 & 2 & 1.51 & 0.57 \\
\hline QAS_L & 1.25 & 2.22 & 1.83 & 0.82 & 0.63 & 1.39 & 1.24 & 0.73 \\
\hline QAS_M & 0.5 & 1.9 & 1.83 & 0.82 & 1.48 & 1.65 & 0.73 & 0.68 \\
\hline TAS_L & 1.82 & 3.01 & 2.4 & 0.86 & 1.46 & 1.97 & 1.32 & 0.76 \\
\hline TAS_U & 1.99 & 3 & 2.24 & 0.87 & 2.48 & 2.86 & 1.43 & 0.74 \\
\hline UPE_L & 3.36 & 4.18 & 2.49 & 0.72 & 2.61 & 3.06 & 1.59 & 0.76 \\
\hline All AWS & 1.66 & 2.8 & 1.98 & 0.79 & 1.2 & 2.28 & 1.43 & 0.74 \\
\hline 17 selected AWS & 1.52 & 2.72 & 1.95 & 0.83 & 1.1 & 2.3 & 1.42 & 0.78 \\
\hline
\end{tabular}

* Dissmissed in the study. see Table S1

Table S17. Same as Table S13 but for ASR. 


\begin{tabular}{|c|c|c|c|c|c|c|c|c|}
\hline \multirow{2}{*}{$\begin{array}{c}\mathrm{W} 10 \mathrm{M}\left(\mathrm{ms}^{-1}\right) \\
\text { E5 }\end{array}$} & \multicolumn{4}{|c|}{ Annually } & \multicolumn{4}{|c|}{ Summer } \\
\hline & $\mathrm{MB}$ & RMSE & RMSEc & $\mathrm{r}$ & MB & RMSE & RMSEc & $\mathrm{r}$ \\
\hline KPC_L & -2.26 & 2.71 & 1.49 & 0.85 & -2.7 & 2.92 & 1.1 & 0.83 \\
\hline KPC_U & 0.45 & 1.18 & 1.08 & 0.9 & 0.3 & 1.07 & 1.02 & 0.88 \\
\hline SCO_L* & -2.22 & 2.51 & 1.19 & 0.59 & -2.02 & 2.32 & 1.14 & 0.65 \\
\hline SCO_U* & -3.43 & 3.66 & 1.3 & 0.65 & -2.78 & 2.96 & 1.03 & 0.61 \\
\hline TAS_A & -2.31 & 3.36 & 2.44 & 0.88 & -1.27 & 1.96 & 1.49 & 0.8 \\
\hline QAS_U & -0.82 & 1.72 & 1.51 & 0.87 & -0.49 & 1.23 & 1.13 & 0.77 \\
\hline QAS_A* & -1.48 & 2.39 & 1.88 & 0.76 & -1.24 & 1.9 & 1.44 & 0.55 \\
\hline NUK_U & -0.65 & 1.62 & 1.49 & 0.86 & 0.17 & 0.99 & 0.98 & 0.79 \\
\hline NUK_N & -1.88 & 2.17 & 1.1 & 0.92 & -1.76 & 1.95 & 0.84 & 0.82 \\
\hline KAN_L & -0.97 & 1.41 & 1.03 & 0.9 & -0.8 & 1.21 & 0.91 & 0.85 \\
\hline KAN_M & 0.84 & 1.7 & 1.48 & 0.9 & 1.99 & 2.37 & 1.29 & 0.8 \\
\hline KAN_U & 0.53 & 1.31 & 1.2 & 0.95 & 0.71 & 1.23 & 1 & 0.91 \\
\hline UPE_U & -0.5 & 1.35 & 1.26 & 0.91 & -0.14 & 1.02 & 1.01 & 0.88 \\
\hline THU_L & -3.14 & 4.08 & 2.6 & 0.8 & -3.71 & 4.32 & 2.21 & 0.92 \\
\hline THU_U & -3.33 & 4.1 & 2.39 & 0.79 & -3.86 & 4.37 & 2.04 & 0.91 \\
\hline NUK_L* & -0.44 & 1.3 & 1.22 & 0.81 & -0.34 & 1.09 & 1.03 & 0.68 \\
\hline QAS_L & -1.15 & 2.1 & 1.76 & 0.78 & -1.38 & 1.97 & 1.4 & 0.54 \\
\hline QAS_M & -1.87 & 2.47 & 1.61 & 0.87 & -1.55 & 1.75 & 0.81 & 0.64 \\
\hline TAS_L & -0.71 & 2.26 & 2.14 & 0.9 & -0.81 & 1.2 & 0.88 & 0.79 \\
\hline TAS_U & -1.37 & 2.49 & 2.08 & 0.91 & -0.81 & 1.25 & 0.96 & 0.81 \\
\hline UPE_L & 0.21 & 1.82 & 1.81 & 0.75 & -0.4 & 1.26 & 1.2 & 0.77 \\
\hline All AWS & -1.08 & 2.15 & 1.55 & 0.83 & -0.95 & 1.89 & 1.19 & 0.78 \\
\hline 17 selected AWS & -0.87 & 2.07 & 1.61 & 0.86 & -0.78 & 1.84 & 1.21 & 0.82 \\
\hline
\end{tabular}

* Dissmissed in the study. see Table S1

Table S18. Same as Table S13 but for ERA5. 


\begin{tabular}{|c|c|c|c|c|c|c|c|c|}
\hline \multirow{2}{*}{$\begin{array}{c}\mathrm{W} 10 \mathrm{M}\left(\mathrm{ms}^{-1}\right) \\
\text { EI }\end{array}$} & \multicolumn{4}{|c|}{ Annually } & \multicolumn{4}{|c|}{ Summer } \\
\hline & $\mathrm{MB}$ & RMSE & RMSEc & $\mathrm{r}$ & MB & RMSE & RMSEc & $\mathrm{r}$ \\
\hline KPC_L & -2.7 & 3.35 & 1.99 & 0.72 & -3.17 & 3.41 & 1.27 & 0.77 \\
\hline KPC_U & 0.28 & 1.27 & 1.23 & 0.85 & -0.18 & 0.98 & 0.97 & 0.86 \\
\hline SCO_L* & -1.56 & 1.88 & 1.04 & 0.65 & -1.6 & 1.89 & 1.01 & 0.69 \\
\hline SCO_U* & -2.39 & 2.64 & 1.11 & 0.67 & -2.09 & 2.28 & 0.91 & 0.63 \\
\hline TAS_A & -1.68 & 3.24 & 2.77 & 0.86 & -0.33 & 1.56 & 1.52 & 0.77 \\
\hline QAS_U & -0.4 & 2.27 & 2.24 & 0.75 & -0.08 & 1.41 & 1.41 & 0.7 \\
\hline QAS_A* & -1.03 & 2.82 & 2.63 & 0.59 & -0.87 & 1.79 & 1.56 & 0.55 \\
\hline NUK_U & -1.2 & 2.1 & 1.72 & 0.81 & -1.37 & 1.83 & 1.21 & 0.67 \\
\hline NUK_N & -1.6 & 2.13 & 1.4 & 0.85 & -1.75 & 2.03 & 1.03 & 0.73 \\
\hline KAN_L & -0.56 & 1.41 & 1.29 & 0.84 & -1.13 & 1.42 & 0.85 & 0.8 \\
\hline KAN_M & -0.38 & 1.41 & 1.36 & 0.9 & 0.11 & 1.21 & 1.21 & 0.78 \\
\hline KAN_U & 0.24 & 1.48 & 1.47 & 0.92 & 0.32 & 1.17 & 1.13 & 0.88 \\
\hline UPE_U & -2.21 & 3 & 2.03 & 0.79 & -1.67 & 2.29 & 1.57 & 0.61 \\
\hline THU_L & -2.71 & 3.6 & 2.37 & 0.82 & -2.99 & 3.75 & 2.26 & 0.88 \\
\hline THU_U & -2.86 & 3.56 & 2.12 & 0.83 & -3.11 & 3.7 & 2.02 & 0.89 \\
\hline NUK_L* & 0.15 & 1.78 & 1.77 & 0.68 & -0.88 & 1.74 & 1.5 & 0.41 \\
\hline QAS_L & -0.46 & 2.13 & 2.08 & 0.74 & -0.67 & 1.65 & 1.51 & 0.61 \\
\hline QAS_M & -1.83 & 2.95 & 2.31 & 0.71 & -0.88 & 1.54 & 1.27 & 0.6 \\
\hline TAS_L & -0.03 & 2.76 & 2.76 & 0.82 & 0.18 & 1.19 & 1.17 & 0.65 \\
\hline TAS_U & -0.73 & 2.75 & 2.65 & 0.84 & 0.13 & 1.16 & 1.15 & 0.72 \\
\hline UPE_L & -0.31 & 2.16 & 2.14 & 0.59 & -0.53 & 1.69 & 1.61 & 0.52 \\
\hline All AWS & -1.03 & 2.28 & 1.82 & 0.78 & -1.06 & 1.88 & 1.33 & 0.71 \\
\hline 17 selected AWS & -0.97 & 2.31 & 1.91 & 0.8 & -0.96 & 1.86 & 1.37 & 0.74 \\
\hline
\end{tabular}

* Dissmissed in the study. see Table S1

Table S19. Same as Table S13 but for ERA-Interim. 


\begin{tabular}{|c|c|c|c|c|c|c|c|c|}
\hline \multirow{2}{*}{$\begin{array}{c}\mathrm{LWD}\left(\mathrm{Wm}^{-2}\right) \\
\text { MAR }_{\mathrm{E} 5}\end{array}$} & \multicolumn{4}{|c|}{ Annually } & \multicolumn{4}{|c|}{ Summer } \\
\hline & MB & RMSE & RMSEc & $\mathrm{r}$ & MB & RMSE & RMSEc & $\mathrm{r}$ \\
\hline KPC_L & -12.82 & 26.84 & 23.58 & 0.87 & -23.63 & 29.57 & 17.79 & 0.73 \\
\hline KPC_U & -8.98 & 25.41 & 23.77 & 0.85 & -13.7 & 23.86 & 19.54 & 0.73 \\
\hline SCO_L* & -25.49 & 34.51 & 23.27 & 0.87 & -40.6 & 44.79 & 18.9 & 0.75 \\
\hline SCO_U & -7.57 & 23.02 & 21.74 & 0.88 & -19.66 & 27.37 & 19.04 & 0.76 \\
\hline TAS_A & -4.62 & 25.65 & 25.23 & 0.77 & -9.91 & 19.66 & 16.98 & 0.81 \\
\hline QAS_U* & -10.41 & 30.48 & 28.65 & 0.81 & -10.5 & 22.25 & 19.61 & 0.79 \\
\hline QAS_A & -10.78 & 26.26 & 23.94 & 0.87 & -11.24 & 20 & 16.54 & 0.85 \\
\hline NUK_U* & -7.2 & 26.1 & 25.08 & 0.86 & -13.51 & 27.45 & 23.9 & 0.73 \\
\hline NUK_N & -10.68 & 24.44 & 21.99 & 0.9 & -15.39 & 22.96 & 17.03 & 0.84 \\
\hline KAN_L & -10.48 & 25.14 & 22.85 & 0.88 & -13.98 & 21.76 & 16.67 & 0.8 \\
\hline KAN_M & -5.68 & 24.09 & 23.41 & 0.87 & -10.31 & 22.35 & 19.83 & 0.8 \\
\hline KAN_U & -5.08 & 23.37 & 22.81 & 0.86 & -10.08 & 23.49 & 21.21 & 0.81 \\
\hline UPE_U & -2.56 & 25.32 & 25.19 & 0.87 & -9.05 & 23.92 & 22.15 & 0.77 \\
\hline THU_L & -18.33 & 30.68 & 24.6 & 0.88 & -20.83 & 26.99 & 17.16 & 0.8 \\
\hline THU_U & -15.15 & 29.39 & 25.18 & 0.88 & -11.89 & 20.05 & 16.14 & 0.85 \\
\hline NUK_L & -20.31 & 31.55 & 24.14 & 0.87 & -24.24 & 30.11 & 17.85 & 0.81 \\
\hline QAS_L & -12.62 & 22.49 & 18.62 & 0.92 & -15.86 & 22.93 & 16.56 & 0.82 \\
\hline QAS_M & -10.58 & 17.66 & 14.14 & 0.96 & -13.15 & 17.44 & 11.46 & 0.93 \\
\hline TAS_L & -10.31 & 26.99 & 24.94 & 0.78 & -14.02 & 22.33 & 17.38 & 0.77 \\
\hline TAS_U* & -9.49 & 28.31 & 26.67 & 0.77 & -16.33 & 28.75 & 23.66 & 0.63 \\
\hline UPE_L & -15.62 & 31 & 26.78 & 0.85 & -17.35 & 27.25 & 21.01 & 0.71 \\
\hline All AWS & -11.28 & 27.06 & 24.05 & 0.86 & -16.43 & 25.84 & 19.17 & 0.77 \\
\hline 17 selected AWS & -10.58 & 26.2 & 23.54 & 0.87 & -15.12 & 24.33 & 18.61 & 0.79 \\
\hline
\end{tabular}

* Dissmissed in the study. see Table S1

Table S20. Annual and summer long wave downward radiation fluxes (LWD) mean bias (MB). RMSE. centered RMSE (RMSEc) and correlation (r) between daily observations at PROMICE AWS and MAR E5 $_{\text {over } 2010-2016 .}$ 


\begin{tabular}{|c|c|c|c|c|c|c|c|c|}
\hline \multirow{2}{*}{$\begin{array}{c}\mathrm{LWD}\left(\mathrm{Wm}^{-2}\right) \\
\text { MAR }_{\mathrm{EI}}\end{array}$} & \multicolumn{4}{|c|}{ Annually } & \multicolumn{4}{|c|}{ Summer } \\
\hline & MB & RMSE & RMSEc & $\mathrm{r}$ & MB & RMSE & RMSEc & $\mathrm{r}$ \\
\hline KPC_L & -12.12 & 25.84 & 22.83 & 0.88 & -20.59 & 27 & 17.46 & 0.75 \\
\hline KPC_U & -7.93 & 24.55 & 23.23 & 0.86 & -10.79 & 21.55 & 18.66 & 0.77 \\
\hline SCO_L* & -25.33 & 34.13 & 22.88 & 0.87 & -38.2 & 42.76 & 19.23 & 0.76 \\
\hline SCO_U & -7.03 & 22.76 & 21.65 & 0.89 & -17.02 & 25.36 & 18.8 & 0.78 \\
\hline TAS_A & -5.74 & 26.26 & 25.63 & 0.77 & -10.26 & 20.59 & 17.85 & 0.79 \\
\hline QAS_U* & -11.36 & 30.93 & 28.77 & 0.81 & -11 & 23 & 20.2 & 0.78 \\
\hline QAS_A & -12.32 & 27.6 & 24.7 & 0.86 & -14.29 & 22.73 & 17.68 & 0.83 \\
\hline NUK_U* & -7.78 & 26.31 & 25.14 & 0.86 & -13.93 & 27.64 & 23.87 & 0.73 \\
\hline NUK_N & -11.01 & 24.4 & 21.77 & 0.9 & -15.43 & 22.86 & 16.87 & 0.84 \\
\hline KAN_L & -10.36 & 24.28 & 21.96 & 0.89 & -14.09 & 21.86 & 16.72 & 0.8 \\
\hline KAN_M & -6.34 & 23.68 & 22.82 & 0.88 & -10.85 & 21.83 & 18.94 & 0.82 \\
\hline KAN_U & -5.66 & 23.12 & 22.41 & 0.87 & -10.73 & 22.79 & 20.1 & 0.83 \\
\hline UPE_U & -5.14 & 24.62 & 24.08 & 0.88 & -10.78 & 23.02 & 20.34 & 0.8 \\
\hline THU_L & -20.74 & 31.59 & 23.82 & 0.89 & -21.93 & 28.1 & 17.57 & 0.78 \\
\hline THU_U & -17.95 & 30.5 & 24.66 & 0.88 & -13.77 & 21.36 & 16.33 & 0.84 \\
\hline NUK_L & -20.31 & 31.39 & 23.93 & 0.87 & -24.06 & 29.79 & 17.56 & 0.82 \\
\hline QAS_L & -13.33 & 23.13 & 18.9 & 0.92 & -16.18 & 23.49 & 17.02 & 0.81 \\
\hline QAS_M & -10.95 & 18.73 & 15.19 & 0.95 & -11.61 & 22.74 & 19.55 & 0.76 \\
\hline TAS_L & -10.56 & 26.63 & 24.45 & 0.79 & -12.92 & 21.5 & 17.19 & 0.78 \\
\hline TAS_U* & -9.88 & 28.21 & 26.42 & 0.78 & -15.43 & 28.54 & 24.01 & 0.62 \\
\hline UPE_L & -18.15 & 31.53 & 25.78 & 0.86 & -19.07 & 27.57 & 19.91 & 0.74 \\
\hline All AWS & -11.98 & 27 & 23.66 & 0.86 & -16.28 & 25.45 & 18.93 & 0.78 \\
\hline 17 selected AWS & -11.35 & 26.11 & 23.08 & 0.87 & -15.11 & 23.93 & 18.22 & 0.8 \\
\hline
\end{tabular}

* Dissmissed in the study. see Table S1

Table S21. Same as Table S20 but for MAR EI $_{\text {. }}$ 


\begin{tabular}{|c|c|c|c|c|c|c|c|c|}
\hline \multirow{2}{*}{$\begin{array}{c}\mathrm{LWD}\left(\mathrm{Wm}^{-2}\right) \\
\mathrm{ASR}\end{array}$} & \multicolumn{4}{|c|}{ Annually } & \multicolumn{4}{|c|}{ Summer } \\
\hline & $\mathrm{MB}$ & RMSE & RMSEc & $\mathrm{r}$ & $\mathrm{MB}$ & RMSE & RMSEc & $\mathrm{r}$ \\
\hline KPC_L & -22.63 & 27.96 & 16.42 & 0.94 & -24.56 & 28.87 & 15.18 & 0.79 \\
\hline KPC_U & -16.81 & 24.6 & 17.96 & 0.92 & -13.15 & 19.94 & 14.99 & 0.83 \\
\hline SCO_L* & -36.05 & 39.69 & 16.59 & 0.93 & -45.81 & 47.86 & 13.87 & 0.83 \\
\hline SCO_U & -22.17 & 26.61 & 14.71 & 0.95 & -29.24 & 32.56 & 14.33 & 0.84 \\
\hline TAS_A & -8.58 & 22.86 & 21.19 & 0.85 & -13.42 & 19.55 & 14.21 & 0.86 \\
\hline QAS_U* & -9.64 & 28.07 & 26.36 & 0.85 & -6.63 & 18.56 & 17.34 & 0.84 \\
\hline QAS_A & -11.63 & 23.82 & 20.79 & 0.91 & -8.53 & 14.55 & 11.8 & 0.92 \\
\hline NUK_U* & -14.37 & 26.68 & 22.48 & 0.9 & -14.9 & 26.54 & 21.96 & 0.76 \\
\hline NUK_N & -14.72 & 22.07 & 16.44 & 0.95 & -8.9 & 16.34 & 13.71 & 0.9 \\
\hline KAN_L & -13.73 & 22.63 & 18 & 0.94 & -5.68 & 14.19 & 13 & 0.88 \\
\hline KAN_M & -12.82 & 22.97 & 19.06 & 0.93 & -5.34 & 16.8 & 15.92 & 0.88 \\
\hline KAN_U & -15.06 & 25.18 & 20.19 & 0.92 & -5.67 & 18.27 & 17.37 & 0.87 \\
\hline UPE_U & -10.15 & 21.51 & 18.96 & 0.93 & -10.29 & 19 & 15.97 & 0.87 \\
\hline THU_L & -18.74 & 27.55 & 20.19 & 0.93 & -11.6 & 19.88 & 16.15 & 0.84 \\
\hline THU_U & -16.51 & 28.29 & 22.97 & 0.92 & -4.02 & 16.05 & 15.54 & 0.86 \\
\hline NUK_L & -23.94 & 32.34 & 21.74 & 0.91 & -18.46 & 24.15 & 15.56 & 0.86 \\
\hline QAS_L & -12.03 & 19.37 & 15.18 & 0.95 & -9.92 & 16.93 & 13.71 & 0.89 \\
\hline QAS_M & -8.02 & 14.58 & 12.17 & 0.97 & -4.05 & 12.26 & 11.58 & 0.92 \\
\hline TAS_L & -14.15 & 24.94 & 20.54 & 0.86 & -13.64 & 20.6 & 15.44 & 0.82 \\
\hline TAS_U* & -12.41 & 25.57 & 22.36 & 0.85 & -15 & 25.58 & 20.71 & 0.71 \\
\hline UPE_L & -26.15 & 34.24 & 22.11 & 0.9 & -22.88 & 28.51 & 17.01 & 0.82 \\
\hline All AWS & -17.06 & 26.59 & 19.6 & 0.91 & -14.81 & 22.78 & 15.83 & 0.84 \\
\hline 17 selected AWS & -16.55 & 25.48 & 18.98 & 0.92 & -12.91 & 20.69 & 15.22 & 0.86 \\
\hline
\end{tabular}

* Dissmissed in the study. see Table S1

Table S22. Same as Table S20 but for ASR. 


\begin{tabular}{|c|c|c|c|c|c|c|c|c|}
\hline \multirow{2}{*}{$\begin{array}{c}\mathrm{LWD}\left(\mathrm{Wm}^{-2}\right) \\
\text { E5 }\end{array}$} & \multicolumn{4}{|c|}{ Annually } & \multicolumn{4}{|c|}{ Summer } \\
\hline & MB & RMSE & RMSEc & $\mathrm{r}$ & $\mathrm{MB}$ & RMSE & RMSEc & $\mathrm{r}$ \\
\hline KPC_L & -18.25 & 23.45 & 14.74 & 0.95 & -23.23 & 27.09 & 13.95 & 0.83 \\
\hline KPC_U & -13.18 & 20.87 & 16.18 & 0.93 & -12.84 & 18.33 & 13.09 & 0.87 \\
\hline SCO_L* & -42.86 & 45.77 & 16.06 & 0.94 & -56.54 & 58.23 & 13.94 & 0.82 \\
\hline SCO_U & -27.09 & 30.63 & 14.31 & 0.95 & -37.94 & 40.37 & 13.81 & 0.85 \\
\hline TAS_A & -2.46 & 17.73 & 17.56 & 0.9 & -1.43 & 13.45 & 13.38 & 0.88 \\
\hline QAS_U* & -15.36 & 29.15 & 24.78 & 0.87 & -9.18 & 18.76 & 16.35 & 0.86 \\
\hline QAS_A & -12.79 & 23.02 & 19.14 & 0.92 & -6.07 & 10.35 & 8.38 & 0.96 \\
\hline NUK_U* & -16.4 & 25.15 & 19.07 & 0.92 & -16.64 & 26.89 & 21.12 & 0.8 \\
\hline NUK_N & -14.97 & 19.99 & 13.24 & 0.97 & -5.55 & 14.08 & 12.94 & 0.92 \\
\hline KAN_L & -6.36 & 18.03 & 16.87 & 0.95 & 7.94 & 14.83 & 12.53 & 0.9 \\
\hline KAN_M & -11.71 & 18.04 & 13.72 & 0.95 & -9.49 & 15.41 & 12.14 & 0.93 \\
\hline KAN_U & -14.18 & 20.45 & 14.73 & 0.94 & -14.12 & 19.25 & 13.09 & 0.93 \\
\hline UPE_U & -11.18 & 19.28 & 15.7 & 0.95 & -5.61 & 15.62 & 14.58 & 0.89 \\
\hline THU_L & -13.98 & 22.58 & 17.73 & 0.94 & -7.12 & 15.38 & 13.64 & 0.89 \\
\hline THU_U & -11.65 & 23.16 & 20.02 & 0.93 & -0.89 & 12.7 & 12.67 & 0.91 \\
\hline NUK_L & -27.32 & 32.72 & 18.01 & 0.93 & -22.48 & 27.21 & 15.32 & 0.89 \\
\hline QAS_L & -13.99 & 19.42 & 13.46 & 0.96 & -3.83 & 13.69 & 13.15 & 0.89 \\
\hline QAS_M & -15.66 & 18.35 & 9.57 & 0.98 & -7.64 & 13.19 & 10.75 & 0.93 \\
\hline TAS_L & -15.8 & 23.85 & 17.87 & 0.9 & -11.17 & 17.12 & 12.97 & 0.89 \\
\hline TAS_U* & -13.02 & 24.55 & 20.81 & 0.87 & -10.7 & 23.21 & 20.6 & 0.73 \\
\hline UPE_L & -27.58 & 33.53 & 19.07 & 0.93 & -17.48 & 23.56 & 15.8 & 0.84 \\
\hline All AWS & -17.2 & 24.99 & 17.04 & 0.93 & -14.22 & 22.35 & 14.38 & 0.87 \\
\hline 17 selected AWS & -15.58 & 23.02 & 16.18 & 0.94 & -11.23 & 19.41 & 13.5 & 0.89 \\
\hline
\end{tabular}

* Dissmissed in the study. see Table S1

Table S23. Same as Table S20 but for ERA5. 


\begin{tabular}{|c|c|c|c|c|c|c|c|c|}
\hline \multirow{2}{*}{$\begin{array}{c}\mathrm{LWD}\left(\mathrm{Wm}^{-2}\right) \\
\mathrm{EI}\end{array}$} & \multicolumn{4}{|c|}{ Annually } & \multicolumn{4}{|c|}{ Summer } \\
\hline & MB & RMSE & RMSEc & $\mathrm{r}$ & $\mathrm{MB}$ & RMSE & RMSEc & $\mathrm{r}$ \\
\hline KPC_L & -13.89 & 21.08 & 15.86 & 0.94 & -23.02 & 26.58 & 13.29 & 0.87 \\
\hline KPC_U & -7.3 & 18.46 & 16.96 & 0.92 & -11.32 & 17.65 & 13.54 & 0.87 \\
\hline SCO_L* & -53.42 & 55.69 & 15.73 & 0.95 & -50.93 & 53.8 & 17.34 & 0.85 \\
\hline SCO_U & -37.11 & 39.66 & 14 & 0.96 & -35.79 & 39.02 & 15.55 & 0.86 \\
\hline TAS_A & -21.87 & 29.28 & 19.47 & 0.89 & -21.79 & 24.57 & 11.36 & 0.92 \\
\hline QAS_U* & -26.96 & 40.13 & 29.73 & 0.84 & -17.27 & 24.6 & 17.52 & 0.83 \\
\hline QAS_A & -25.16 & 36.4 & 26.3 & 0.88 & -14.31 & 20.55 & 14.75 & 0.88 \\
\hline NUK_U* & -8.25 & 22.57 & 21.01 & 0.9 & -16.31 & 27.17 & 21.74 & 0.75 \\
\hline NUK_N & -10.65 & 17.34 & 13.69 & 0.96 & -12.24 & 18.56 & 13.95 & 0.88 \\
\hline KAN_L & -16.09 & 24.27 & 18.17 & 0.94 & -10.39 & 17.98 & 14.68 & 0.88 \\
\hline KAN_M & -10.5 & 19.33 & 16.22 & 0.95 & -7.84 & 16.4 & 14.4 & 0.9 \\
\hline KAN_U & -11.73 & 21.27 & 17.75 & 0.93 & -8.9 & 18.93 & 16.71 & 0.89 \\
\hline UPE_U & -9.82 & 21.21 & 18.8 & 0.94 & -1.23 & 15.36 & 15.31 & 0.89 \\
\hline THU_L & -23.87 & 38.1 & 29.69 & 0.89 & -4.41 & 17.46 & 16.89 & 0.79 \\
\hline THU_U & -20.01 & 38.19 & 32.53 & 0.87 & 3.16 & 17.68 & 17.4 & 0.8 \\
\hline NUK_L & -23.72 & 30.73 & 19.54 & 0.91 & -30.82 & 34.58 & 15.69 & 0.84 \\
\hline QAS_L & -31.03 & 35.14 & 16.48 & 0.95 & -26.65 & 30.42 & 14.67 & 0.87 \\
\hline QAS_M & -28.92 & 32.83 & 15.54 & 0.96 & -25.19 & 28.33 & 12.97 & 0.91 \\
\hline TAS_L & -34.91 & 40.22 & 19.97 & 0.88 & -31.96 & 35.04 & 14.36 & 0.86 \\
\hline TAS_U* & -32.24 & 39.09 & 22.11 & 0.87 & -30.55 & 36.11 & 19.25 & 0.77 \\
\hline UPE_L & -26.41 & 33.97 & 21.36 & 0.92 & -17.22 & 23.76 & 16.38 & 0.84 \\
\hline All AWS & -22.16 & 30.9 & 19.88 & 0.92 & -18.57 & 26.1 & 15.9 & 0.85 \\
\hline 17 selected AWS & -19.6 & 28.28 & 19.26 & 0.92 & -15.58 & 23.39 & 15.11 & 0.86 \\
\hline
\end{tabular}

* Dissmissed in the study. see Table S1

Table S24. Same as Table S20 but for ERA-Interim. 


\begin{tabular}{|c|c|c|c|c|c|c|c|c|}
\hline \multirow{2}{*}{$\begin{array}{c}\mathrm{SWD}\left(\mathrm{Wm}^{-2}\right) \\
\text { MAR }_{\mathrm{E} 5}\end{array}$} & \multicolumn{4}{|c|}{ Annually } & \multicolumn{4}{|c|}{ Summer } \\
\hline & $\mathrm{MB}$ & RMSE & RMSEc & $\mathrm{r}$ & MB & RMSE & RMSEc & $\mathrm{r}$ \\
\hline KPC_L & -3.27 & 30.54 & 30.36 & 0.97 & 2.26 & 48.18 & 48.13 & 0.89 \\
\hline KPC_U & -11.5 & 28.3 & 25.86 & 0.98 & -14.83 & 38.59 & 35.63 & 0.93 \\
\hline SCO_L* & 8.35 & 31.27 & 30.13 & 0.97 & 27.16 & 52.61 & 45.06 & 0.87 \\
\hline SCO_U & -2.28 & 28.57 & 28.48 & 0.98 & 10.29 & 43.41 & 42.18 & 0.89 \\
\hline TAS_A & -8.94 & 36.08 & 34.96 & 0.96 & -12.56 & 40.15 & 38.14 & 0.89 \\
\hline QAS_U* & 18.43 & 82.13 & 80.04 & 0.79 & 15.28 & 100.55 & 99.39 & 0.53 \\
\hline QAS_A & -1.85 & 37.63 & 37.58 & 0.95 & -17.33 & 44.57 & 41.06 & 0.89 \\
\hline NUK_U* & 3.73 & 69.2 & 69.1 & 0.83 & 22.81 & 102.58 & 100.02 & 0.57 \\
\hline NUK_N & -3.46 & 30.15 & 29.95 & 0.97 & 3.44 & 45.28 & 45.15 & 0.88 \\
\hline KAN_L & -14.09 & 35.87 & 32.99 & 0.96 & -8.88 & 45.69 & 44.82 & 0.85 \\
\hline KAN_M & -10.74 & 30.84 & 28.91 & 0.97 & -7.05 & 40.77 & 40.15 & 0.89 \\
\hline KAN_U & -20.36 & 36.79 & 30.64 & 0.98 & -22.97 & 45.45 & 39.22 & 0.86 \\
\hline UPE_U & -11.66 & 33.23 & 31.12 & 0.97 & -16.85 & 51.71 & 48.89 & 0.87 \\
\hline THU_L & -4.32 & 28.61 & 28.28 & 0.97 & 4.77 & 43.52 & 43.26 & 0.9 \\
\hline THU_U & -5.16 & 25.92 & 25.4 & 0.98 & -4.62 & 38.47 & 38.19 & 0.92 \\
\hline NUK_L* & 10.08 & 52.62 & 51.65 & 0.9 & 14 & 62.61 & 61.03 & 0.8 \\
\hline QAS_L & 1.88 & 35.36 & 35.31 & 0.95 & 16.99 & 54.57 & 51.86 & 0.85 \\
\hline QAS_M & -2.21 & 24.14 & 24.04 & 0.95 & 23.56 & 46.29 & 39.84 & 0.79 \\
\hline TAS_L & -5.4 & 40.41 & 40.05 & 0.95 & 0.7 & 50.91 & 50.91 & 0.84 \\
\hline TAS_U* & 4.73 & 72.2 & 72.04 & 0.81 & 25.76 & 113.4 & 110.44 & 0.39 \\
\hline UPE_L & -6.88 & 36.73 & 36.08 & 0.95 & -2.3 & 54.83 & 54.78 & 0.83 \\
\hline All AWS & -3.17 & 40.47 & 39.12 & 0.94 & 2.43 & 56.35 & 54.26 & 0.81 \\
\hline 16 selected AWS & -7.95 & 32.8 & 31.3 & 0.97 & -4.42 & 46.07 & 44.41 & 0.88 \\
\hline
\end{tabular}

* Dissmissed in the study. see Table S1

Table S25. Annual and summer short wave downward radiation fluxes (SWD) mean bias (MB). RMSE. centered RMSE (RMSEc) and correlation (r) between daily observations at PROMICE AWS and MAR E5 over 2010 - 2016. 


\begin{tabular}{|c|c|c|c|c|c|c|c|c|}
\hline \multirow{2}{*}{$\begin{array}{c}\mathrm{SWD}\left(\mathrm{Wm}^{-2}\right) \\
\mathrm{MAR}_{\mathrm{EI}}\end{array}$} & \multicolumn{4}{|c|}{ Annually } & \multicolumn{4}{|c|}{ Summer } \\
\hline & MB & RMSE & RMSEc & $\mathrm{r}$ & MB & RMSE & RMSEc & $\mathrm{r}$ \\
\hline KPC_L & -3.85 & 29.26 & 29 & 0.98 & -0.61 & 45.95 & 45.95 & 0.9 \\
\hline KPC_U & -12.48 & 28.52 & 25.64 & 0.99 & -17.94 & 38.88 & 34.49 & 0.93 \\
\hline SCO_L* & 7.67 & 30.42 & 29.44 & 0.97 & 24.58 & 50.27 & 43.85 & 0.88 \\
\hline SCO_U & -3.32 & 28.09 & 27.89 & 0.98 & 7.17 & 41.38 & 40.75 & 0.9 \\
\hline TAS_A & -8.17 & 36.5 & 35.57 & 0.96 & -12.08 & 42.47 & 40.72 & 0.87 \\
\hline QAS_U* & 18.79 & 82.88 & 80.72 & 0.78 & 15.41 & 101.77 & 100.6 & 0.52 \\
\hline QAS_A & -0.42 & 37.86 & 37.85 & 0.95 & -12.51 & 44.75 & 42.96 & 0.87 \\
\hline NUK_U* & 4.49 & 69.84 & 69.7 & 0.83 & 24.91 & 103.14 & 100.09 & 0.57 \\
\hline NUK_N & -2.94 & 30.61 & 30.47 & 0.97 & 3.86 & 46.56 & 46.4 & 0.87 \\
\hline KAN_L & -13.73 & 36.25 & 33.54 & 0.96 & -7.33 & 46.8 & 46.22 & 0.84 \\
\hline KAN_M & -10.17 & 30.79 & 29.06 & 0.97 & -6.05 & 41.05 & 40.6 & 0.88 \\
\hline KAN_U & -19.69 & 35.75 & 29.84 & 0.98 & -22.08 & 43.75 & 37.77 & 0.88 \\
\hline UPE_U & -9.59 & 30.62 & 29.08 & 0.97 & -11.84 & 46.17 & 44.63 & 0.89 \\
\hline THU_L & -1.85 & 30.31 & 30.25 & 0.97 & 10.01 & 48.11 & 47.05 & 0.88 \\
\hline THU_U & -2.23 & 25.75 & 25.65 & 0.98 & 2.5 & 39.2 & 39.12 & 0.91 \\
\hline NUK_L* & 10.43 & 53.2 & 52.17 & 0.9 & 15.74 & 63.29 & 61.3 & 0.79 \\
\hline QAS_L & 2.38 & 36.88 & 36.81 & 0.95 & 17.82 & 57.99 & 55.19 & 0.83 \\
\hline QAS_M & -2.89 & 23.43 & 23.26 & 0.95 & 20.51 & 48.35 & 43.79 & 0.75 \\
\hline TAS_L & -5.14 & 40.02 & 39.69 & 0.95 & 0.01 & 51.1 & 51.1 & 0.84 \\
\hline TAS_U* & 5.6 & 72.09 & 71.87 & 0.82 & 26.45 & 113.52 & 110.4 & 0.4 \\
\hline UPE_L & -4.67 & 35.18 & 34.87 & 0.96 & 3.25 & 51.27 & 51.17 & 0.85 \\
\hline All AWS & -2.54 & 40.31 & 39.06 & 0.94 & 3.57 & 56.11 & 54.1 & 0.82 \\
\hline 16 selected AWS & -7.18 & 32.52 & 31.15 & 0.97 & -2.98 & 45.74 & 44.18 & 0.88 \\
\hline
\end{tabular}

* Dissmissed in the study. see Table S1

Table S26. Same as Table S25 but for MAR EI $_{\text {. }}$ 


\begin{tabular}{|c|c|c|c|c|c|c|c|c|}
\hline \multirow{2}{*}{$\begin{array}{c}\mathrm{SWD}\left(\mathrm{Wm}^{-2}\right) \\
\mathrm{ASR}\end{array}$} & \multicolumn{4}{|c|}{ Annually } & \multicolumn{4}{|c|}{ Summer } \\
\hline & $\mathrm{MB}$ & RMSE & RMSEc & $\mathrm{r}$ & $\mathrm{MB}$ & RMSE & RMSEc & $\mathrm{r}$ \\
\hline KPC_L & 8.37 & 30.92 & 29.76 & 0.98 & 28.34 & 54.93 & 47.06 & \\
\hline KPC_U & 0.41 & 19.33 & 19.33 & 0.99 & 8.21 & 32.14 & 31.07 & \\
\hline SCO_L* & 24.16 & 43.28 & 35.91 & 0.97 & 56.49 & 73.35 & 46.78 & \\
\hline SCO_U & 15.56 & 35.48 & 31.89 & 0.98 & 43.26 & 62.62 & 45.28 & \\
\hline TAS_A & 9.96 & 33.74 & 32.24 & 0.97 & 23.58 & 46.25 & 39.78 & \\
\hline QAS_U* & 36.63 & 89.5 & 81.66 & 0.79 & 50.99 & 109.18 & 96.54 & \\
\hline QAS_A & 11.76 & 39.54 & 37.74 & 0.95 & 16.83 & 42.76 & 39.31 & \\
\hline NUK_U* & 24.27 & 78.2 & 74.34 & 0.83 & 63.55 & 120.6 & 102.5 & \\
\hline NUK_N & 6.5 & 27.55 & 26.77 & 0.98 & 15.43 & 43.24 & 40.4 & \\
\hline KAN_L & -1.5 & 30.34 & 30.3 & 0.97 & 10.13 & 44.03 & 42.85 & \\
\hline KAN_M & 4.28 & 24.96 & 24.59 & 0.98 & 15.93 & 41.34 & 38.14 & \\
\hline KAN_U & -3.64 & 22.6 & 22.3 & 0.99 & -0.93 & 31.9 & 31.88 & \\
\hline UPE_U & 5.71 & 25.17 & 24.51 & 0.98 & 21.35 & 44.95 & 39.55 & \\
\hline THU_L & 4.69 & 29.34 & 28.96 & 0.97 & 24.34 & 49.87 & 43.53 & \\
\hline THU_U & 6.86 & 26.03 & 25.1 & 0.98 & 20.95 & 42.89 & 37.43 & \\
\hline NUK_L* & 21.71 & 57.4 & 53.14 & 0.9 & 33.61 & 69.51 & 60.84 & \\
\hline QAS_L & 15.18 & 39.1 & 36.03 & 0.96 & 41.65 & 64.87 & 49.73 & \\
\hline QAS_M & 2.93 & 27.06 & 26.9 & 0.94 & 37.02 & 54.72 & 40.3 & \\
\hline TAS_L & 17.35 & 48.29 & 45.06 & 0.94 & 41.8 & 72.35 & 59.05 & \\
\hline TAS_U* & 25.96 & 77.9 & 73.45 & 0.83 & 65.27 & 124.15 & 105.61 & \\
\hline UPE_L & 10.75 & 36.1 & 34.47 & 0.97 & 34.36 & 58.46 & 47.29 & \\
\hline All AWS & 12.28 & 40.84 & 38.39 & 0.94 & 31.1 & 61.83 & 52.27 & \\
\hline 16 selected AWS & 6.8 & 30.31 & 29.09 & 0.97 & 22.87 & 48.69 & 41.88 & \\
\hline
\end{tabular}

* Dissmissed in the study. see Table S1

Table S27. Same as Table S25 but for ASR. 


\begin{tabular}{|c|c|c|c|c|c|c|c|c|}
\hline \multirow{2}{*}{$\begin{array}{c}\mathrm{SWD}\left(\mathrm{Wm}^{-2}\right) \\
\text { E5 }\end{array}$} & \multicolumn{4}{|c|}{ Annually } & \multicolumn{4}{|c|}{ Summer } \\
\hline & $\mathrm{MB}$ & RMSE & RMSEc & $\mathrm{r}$ & MB & RMSE & RMSEc & $\mathrm{r}$ \\
\hline KPC_L & 4.03 & 25.39 & 25.07 & 0.98 & 15.16 & 44.11 & 41.42 & 0.92 \\
\hline KPC_U & -4.15 & 17.9 & 17.41 & 0.99 & -4.28 & 28.25 & 27.92 & 0.96 \\
\hline SCO_L* & 22.78 & 39.57 & 32.36 & 0.98 & 53.75 & 67.8 & 41.33 & 0.89 \\
\hline SCO_U & 13.47 & 30.98 & 27.9 & 0.98 & 38.52 & 55.3 & 39.67 & 0.9 \\
\hline TAS_A & -3.09 & 30.28 & 30.12 & 0.97 & -9.24 & 34.19 & 32.92 & 0.92 \\
\hline QAS_U* & 28.26 & 83.84 & 78.93 & 0.79 & 28.35 & 100.19 & 96.1 & 0.54 \\
\hline QAS_A & 4.22 & 35.82 & 35.57 & 0.95 & -13.05 & 34.85 & 32.31 & 0.93 \\
\hline NUK_U* & 18.15 & 71.43 & 69.09 & 0.84 & 47.39 & 109.16 & 98.34 & 0.57 \\
\hline NUK_N & -9.54 & 26.48 & 24.7 & 0.98 & -25.9 & 43.44 & 34.87 & 0.93 \\
\hline KAN_L & -21.61 & 38.5 & 31.86 & 0.97 & -39.79 & 57.22 & 41.12 & 0.88 \\
\hline KAN_M & 0.78 & 22.16 & 22.15 & 0.98 & 9.27 & 34.33 & 33.05 & 0.93 \\
\hline KAN_U & -9.86 & 22.72 & 20.47 & 0.99 & -12.7 & 30.38 & 27.6 & 0.93 \\
\hline UPE_U & -0.88 & 22.27 & 22.25 & 0.99 & -1.95 & 38.4 & 38.35 & 0.92 \\
\hline THU_L & -10.6 & 27.03 & 24.86 & 0.98 & -18.6 & 41.6 & 37.21 & 0.93 \\
\hline THU_U & -9.48 & 27.1 & 25.39 & 0.98 & -22.48 & 43.87 & 37.68 & 0.92 \\
\hline NUK_L* & 18.84 & 51.92 & 48.39 & 0.91 & 26.26 & 59.94 & 53.88 & 0.83 \\
\hline QAS_L & 1.13 & 27.4 & 27.38 & 0.97 & -1.63 & 43.45 & 43.42 & 0.9 \\
\hline QAS_M & 1.4 & 17.49 & 17.43 & 0.97 & 17.95 & 33.88 & 28.74 & 0.91 \\
\hline TAS_L & 4.53 & 34.33 & 34.03 & 0.96 & 14.07 & 45.02 & 42.76 & 0.89 \\
\hline TAS_U* & 13.96 & 70.44 & 69.04 & 0.83 & 37.04 & 109.9 & 103.47 & 0.46 \\
\hline UPE_L & 2.04 & 27.48 & 27.4 & 0.97 & 4.65 & 43.02 & 42.76 & 0.89 \\
\hline All AWS & 3.56 & 36.86 & 34.73 & 0.95 & 7.83 & 54.01 & 47.71 & 0.85 \\
\hline 16 selected AWS & -2.98 & 26.98 & 25.59 & 0.98 & -3.68 & 41.53 & 37.1 & 0.91 \\
\hline
\end{tabular}

Table S28. Same as Table S25 but for ERA5. 


\begin{tabular}{|c|c|c|c|c|c|c|c|c|}
\hline \multirow{2}{*}{$\begin{array}{c}\mathrm{SWD}\left(\mathrm{Wm}^{-2}\right) \\
\text { EI }\end{array}$} & \multicolumn{4}{|c|}{ Annually } & \multicolumn{4}{|c|}{ Summer } \\
\hline & MB & RMSE & RMSEc & $\mathrm{r}$ & MB & RMSE & RMSEc & $\mathrm{r}$ \\
\hline KPC_L & -0.99 & 23.26 & 23.24 & 0.99 & 7.26 & 35.22 & 34.46 & 0.94 \\
\hline KPC_U & -8.39 & 23.2 & 21.63 & 0.99 & -10.4 & 33.82 & 32.18 & 0.95 \\
\hline SCO_L* & 17.19 & 34.37 & 29.76 & 0.98 & 45.01 & 58.52 & 37.4 & 0.91 \\
\hline SCO_U & 7.8 & 27.91 & 26.79 & 0.98 & 30.74 & 47.71 & 36.48 & 0.92 \\
\hline TAS_A & -3.7 & 30.47 & 30.25 & 0.97 & 1.38 & 31.82 & 31.79 & 0.93 \\
\hline QAS_U* & 19.14 & 81.29 & 79 & 0.79 & 20.33 & 98.24 & 96.11 & 0.55 \\
\hline QAS_A & -4.75 & 37.01 & 36.7 & 0.95 & -16.85 & 41.48 & 37.91 & 0.91 \\
\hline NUK_U* & 3.76 & 69.27 & 69.16 & 0.83 & 18.31 & 103.97 & 102.35 & 0.54 \\
\hline NUK_N & -4.75 & 25.79 & 25.35 & 0.98 & -2.45 & 39.31 & 39.24 & 0.91 \\
\hline KAN_L & -12.55 & 30.8 & 28.12 & 0.97 & -9.16 & 40.07 & 39.01 & 0.9 \\
\hline KAN_M & -6.16 & 27.39 & 26.69 & 0.98 & 2.19 & 38.14 & 38.08 & 0.9 \\
\hline KAN_U & -12.45 & 28.6 & 25.75 & 0.98 & -9.23 & 37.08 & 35.91 & 0.91 \\
\hline UPE_U & -9.92 & 26.67 & 24.75 & 0.98 & -14.68 & 38.95 & 36.08 & 0.93 \\
\hline THU_L & -16.33 & 33.07 & 28.75 & 0.97 & -28.52 & 50.01 & 41.08 & 0.91 \\
\hline THU_U & -16.14 & 34.54 & 30.53 & 0.97 & -35.56 & 55.51 & 42.63 & 0.9 \\
\hline NUK_L* & 10.19 & 51.54 & 50.52 & 0.9 & 10.24 & 59.55 & 58.66 & 0.8 \\
\hline QAS_L & 6.93 & 33.48 & 32.75 & 0.96 & 29.16 & 52.8 & 44.02 & 0.9 \\
\hline QAS_M & -4.24 & 21.82 & 21.4 & 0.96 & 24.1 & 40.03 & 31.96 & 0.9 \\
\hline TAS_L & 5.72 & 36.83 & 36.38 & 0.96 & 26.23 & 51.41 & 44.22 & 0.88 \\
\hline TAS_U* & 13.7 & 73.72 & 72.43 & 0.83 & 47.06 & 116.75 & 106.84 & 0.46 \\
\hline UPE_L & -4.44 & 28.05 & 27.69 & 0.97 & -1.41 & 39.63 & 39.61 & 0.92 \\
\hline All AWS & -0.41 & 38.02 & 36.36 & 0.95 & 6.5 & 53.91 & 48.91 & 0.85 \\
\hline 16 selected AWS & -5.55 & 29.21 & 27.67 & 0.98 & -1.4 & 42.34 & 38.22 & 0.91 \\
\hline
\end{tabular}

* Dissmissed in the study. see Table S1

Table S29. Same as Table S25 but for ERA-Interim. 